\title{
Reviving WHAAM! a comparative evaluation of cleaning systems for the conservation treatment of Roy Lichtenstein's iconic painting
}

\author{
Angelica Bartoletti ${ }^{1,2}$, Rachel Barker ${ }^{1,3}$, David Chelazzi ${ }^{4}$, Nicole Bonelli ${ }^{4}$, Piero Baglioni ${ }^{4}$, Judith Lee ${ }^{1}$, \\ Lora V. Angelova ${ }^{1,5}$ and Bronwyn Ormsby ${ }^{1 *}$ (D)
}

\begin{abstract}
Roy Lichtenstein's Whaam! (1963) is an iconic artwork in Tate's collection (T00897). Over the past 50 years, the painting has been on almost continuous display and had accrued a layer of deposited soiling, which resulted in the dampening of Lichtenstein's vibrant colours and the masking of numerous subtleties across the painting surface. This paper outlines the design and execution of an optimal soiling removal strategy for this challenging work; utilising collaborative, practice-based research. The conservation treatment employed was derived through an iterative process that reflected and supported the conservation decision-making process. The research strands included: technical and art historical investigations to determine the materials and construction of Whaam! and to define the aims of the conservation treatment; preparation of accelerated aged and artificially soiled test (mock-up) paint samples based on contemporary equivalent materials and a comparative evaluation of a range of established and novel soil-removal systems, followed by further tailoring for use on the work of art. The range of cleaning systems evaluated included free-solvents, gels and emulsifiers; which were documented using star diagrams, digital microscopy and infrared spectroscopy. After a rigorous process of assessment and refinement, the strategy taken forward to Whaam! included the use of a polyvinyl alcohol-based polymeric hydrogel (Nanorestore Gel ${ }^{\circledR}$ Peggy 6), uploaded with tailored aqueous solutions. This process facilitated a low risk, controlled and even-removal of the soiling layer, enabling the successful treatment of this sensitive painting for the first time in the painting's history.
\end{abstract}

Keywords: Cleaning, Evaluation, Hydrogel, Emulsifier, Acrylic, Magna, Oil, Lichtenstein, Peggy, Nanorestore, Whaam!

\section{Introduction}

Modern and contemporary painted art can prove challenging with respect to the removal of deposited soiling from paint surfaces, and particularly so for works of art that are displayed unglazed. Furthermore, the use of mixed-media or several paint types in one artwork can demand the tailoring of approaches to cleaning. Conservation treatments carry considerable risk around key aspects such as surface integrity and authenticity, and interactions with the painting surface can involve

\footnotetext{
${ }^{*}$ Correspondence: bronwyn.ormsby@tate.org.uk

${ }^{1}$ Conservation Department, Tate, Millbank, London SW1P 4RG, UK

Full list of author information is available at the end of the article
}

unacceptable pigment pickup, paint swelling, uneven soil removal and gloss change. Over the past decade, research efforts have contributed to the evaluation and refinement of cleaning methods for unprotected painted works of art, resulting in a more nuanced approach and lowerrisk options for soiling removal. Part of this has involved the refinement and tailoring of cleaning approaches to specific paint types and the introduction of novel systems for use on these often unpredictable and challenging paint surfaces. This study aims to contribute to this ongoing endeavour through; the characterisation of the paint types present in Whaam!, reflections on the aims of the cleaning treatment, and an extensive comparative
Springer Open

(c) The Author(s) 2020. This article is licensed under a Creative Commons Attribution 4.0 International License, which permits use, sharing adaptation, distribution and reproduction in any medium or format, as long as you give appropriate credit to the original author(s) and the source, provide a link to the Creative Commons licence, and indicate if changes were made. The images or other third party material in this article are included in the article's Creative Commons licence, unless indicated otherwise in a credit line to the material. If material is not included in the article's Creative Commons licence and your intended use is not permitted by statutory regulation or exceeds the permitted use, you will need to obtain permission directly from the copyright holder. To view a copy of this licence, visit http://creativeco mmons.org/licenses/by/4.0/. The Creative Commons Public Domain Dedication waiver (http://creativecommons.org/publicdomain/ zero/1.0/) applies to the data made available in this article, unless otherwise stated in a credit line to the data. 


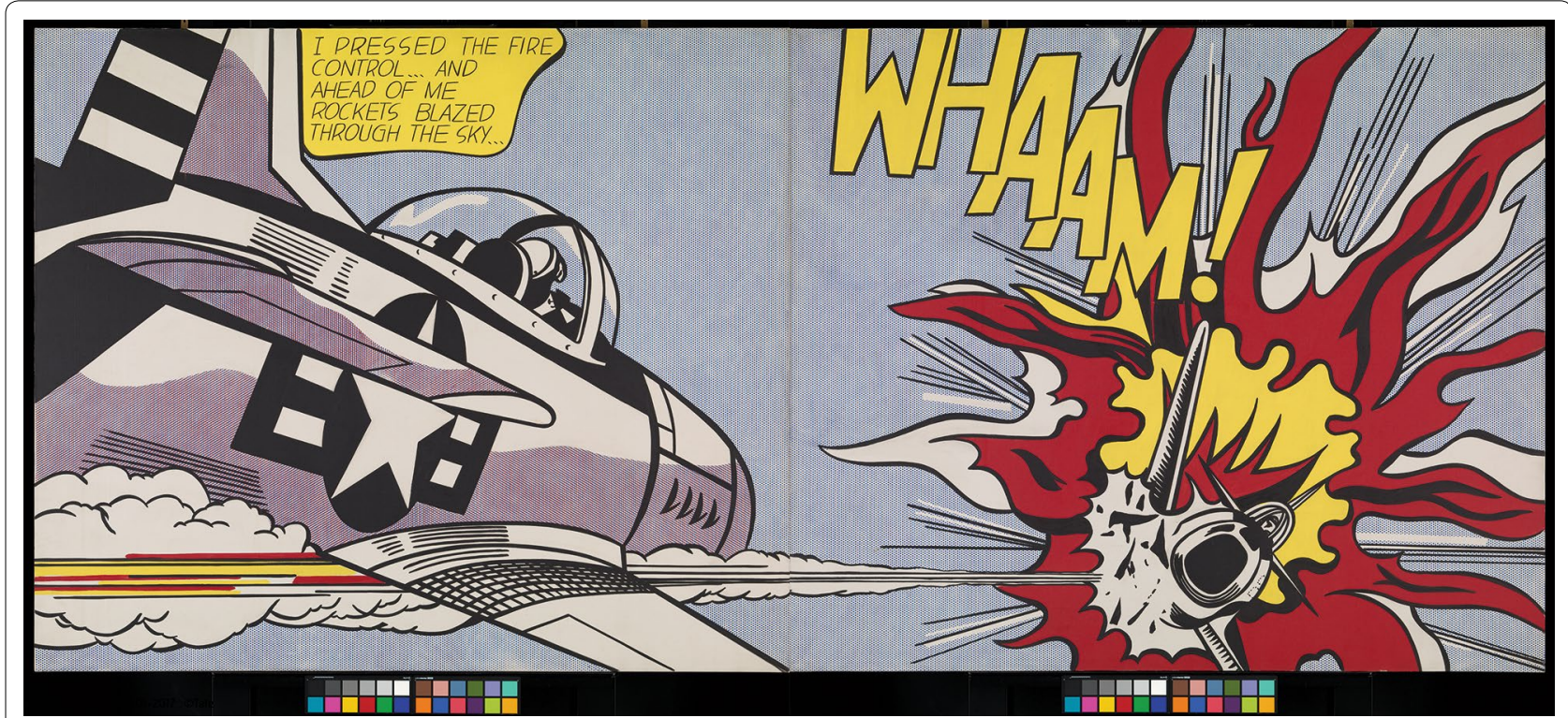

Fig. 1 Roy Lichtenstein, Whaam! 1963. Magna and oil paints on two oil-modified alkyd-primed canvases, $1727 \times 4064$ mm, Tate T00897; Photo @Tate 2017, before cleaning; @The estate of Roy Lichtenstein

study of a range of established and novel soiling removal systems, which were optimised for the each of the paint types present. The supporting research is presented as iterative stages which directly reflect the conservation treatment decision-making process, alongside a detailed account of the treatment carried out.

\section{Whaam!}

Whaam! (Fig. 1) by Roy Lichtenstein (1923-1997) is comprised of two large panels (measuring approximately $1.70 \times 2 \mathrm{~m}$ each), depicting a revisited comic strip from "Star Jockey" by Irv Novick, published in the DC Comics Inc. comic All American Men of War [1, 2]. The work is painted on a commercially primed cotton canvas using three different paints [2]. Bocour Magna acrylic solution, oil-modified alkyd and drying oil; as inscriptions on the back of both panels reveal. Tate purchased the painting in 1966 from Leo Castelli (New York) and despite an initial controversy surrounding its acquisition [3], Whaam! has enduring iconic status within Tate's collection.

The painting is intentionally not varnished; is displayed without a frame ${ }^{1}$ and is unprotected by glazing as per Roy

\footnotetext{
${ }^{1}$ Whaam! has an original wood batten frame, which was removed soon after the painting acquisition and is now archived at Tate Stores.

2 'I like the way the different colours might have little differences in gloss. I'd be against it unless it was very important as a measure to preserve it. If it wasn't highly reflective or even slightly reflective, it would probably be alright but only if it was really necessary and didn't disturb the surface much. MOMA [The Museum of Modern Art] framed my painting of the artists' studio and to me it looks just like a poster. The glazing took away what paint quality there is, you couldn't tell that it wasn't printed on
}

Lichtenstein's wishes. ${ }^{2}$ By 2017, a smooth, almost homogenous layer of glossy, yellowed, greasy and particulate material had deposited onto the painting surface, resulting in significantly compromised visual impact. The white areas appeared a light brown-grey, the brighter colours were uniformly dampened, and numerous subtle features of the paint surface, such as gloss variance, pencil lines and brushstrokes had become obscured. Along the edges of the two large canvases, as is commonly noted with large, unframed works from this period, finger marks and scuffs were also apparent (Fig. 2). In an interview held in 1992 with Jo Crook [4], Lichtenstein revealed that he was aware his paintings attracted dirt. ${ }^{3}$ Whilst Lichtenstein was not necessarily interested in the technicalities of dirt removal, it can be assumed from these encounters that he did not welcome the changes caused by (permanent) dirt deposition.

Pop Art aimed to utilise images of popular culture in art, where aspects of advertising and consumerism were

\section{Footnote 2 (continued)}

paper. I didn't have anything to do with the selection of the frame which is a plain, fairly heavy, flat, natural wood. Excerpt from Tate Public Records: Research: Artists: Conservation interviews TG 23/1/1/111, Jo Crook interview with Roy Lichtenstein, New York studio 26th May 1992.

3 'We haven't had to do anything beyond erasing. No serious cleaning has been carried out by myself or assistants. We have some paintings that have just developed dirt which I don't want to get into cleaning. These are works that were painted some time ago and have developed a surface covering of dirt.' Ibid. 


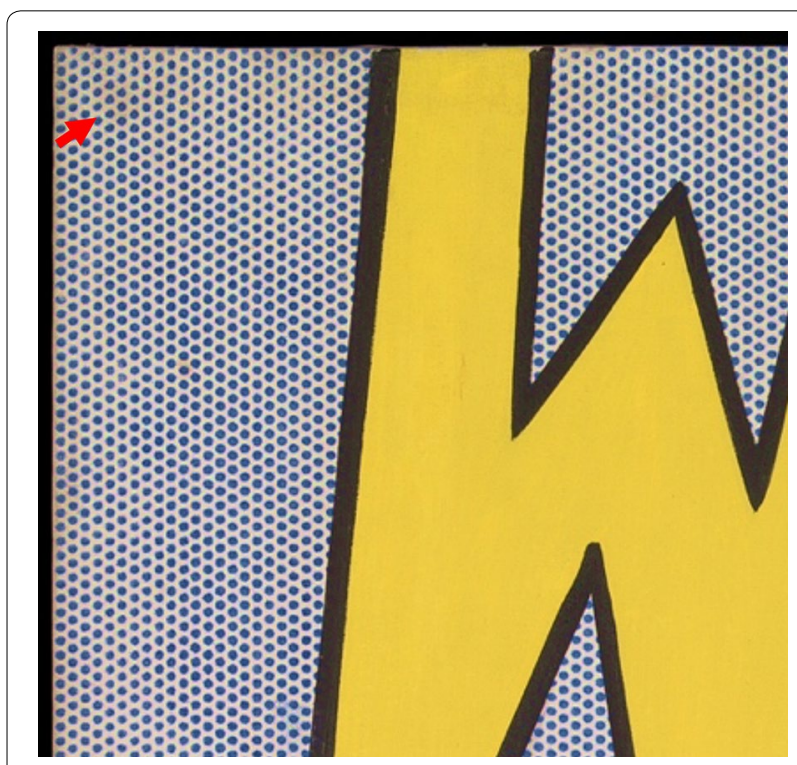

Fig. 2 Detail of finger mark in top left corner of the Ben-Day dot section of the right-hand panel (highlighted by red arrow)

often represented by ironic and superficial imagery. Pop artists appropriated these images and elevated them into a high art form, while often using traditional painting methods. The paintings had to appear as immediate, freshly produced and as culturally relevant as their source material. Accumulations of dirt, finger marks and evidence of wear and tear such as scuff marks, all shroud or distract from the intended dynamism of these paintings. Lichtenstein himself was often quoted as desiring the purest and sharpest colour values. Referring to colour, he said: 'I always painted on a printed ground, but later I painted it again with my own white. I thought the canvas might change colour in time, and also, it's easier to make corrections when I paint on my own white. But more important, I like it whiter now. Everything became sharper, I guess, as I went along and I just like that. ${ }^{4}$

It was thus evident that Whaam! would benefit from surface cleaning treatment to help re-establish Lichtenstein's desired surface aesthetic, to minimise risks associated with the embedding of soiling into painted surfaces $[5,6]$, and to reinstate a more direct viewing experience.

Previous attempts at surface cleaning had been unsuccessful due to the inherent complexity of this work. Whaam! is challenging due to the range of materials Lichtenstein used, including three paint types: oil-modified alkyd, oil, and acrylic solution (Magna), see Table 1,

\footnotetext{
${ }^{4}$ Diane Waldman. 'Roy Lichtenstein Interviewed', in John Coplans (ed.), Roy Lichtenstein, London 1973, p. 112. This is an extract from an interview originally published in: Diane Waldman, Roy Lichtenstein (New York: Abrams, 1971).
}

which each display inherently different sensitivities to solvents. In addition, as noted in an early Whaam! conservation report [7], the painting also presents an overall fragility to mechanical action; where the application of sponges or the gentle rolling motion of a cotton swab can cause unacceptable pigment transfer and changes in surface gloss. The use of dry-cleaning methods has also been reported as inadequate with respect to the desired cleaning efficacy [7], and there is little research on the appropriate use of solvents for the surface cleaning of Magna paints.

The primary objectives of the conservation treatment were to evenly remove the layer of deposited soiling and to reduce the distracting marks scattered around the edges of both canvases while maintaining the overall tonal balance of the work, and minimising any risks associated with using cleaning systems on each paint type. Although a considerable amount is known about Whaam!'s construction [2], further investigations into Lichtenstein's working practices and aesthetic were combined with technical and analytical studies to inform the production of mock-up samples and the desired treatment outcomes.

\section{Whaam! materials and construction}

Whaam! consists of a commercially double-primed cotton duck medium-weight plain-weave canvas, preprimed with an animal glue sizing layer and an oilmodified alkyd (hereafter referred to as alkyd) priming containing titanium white pigment (PW6) with gypsum and silica extenders (Table 1). This relatively lean layer is exposed in several areas, thus functioning as the 'white' parts of the composition. Using a Postoscope projector [2], Lichtenstein projected the drawing ${ }^{5}$ onto the alkydprimed canvas and transferred it using a graphite pencil, ${ }^{6}$ which remains visible on many parts of the painting.

The blue (synthetic ultramarine blue, PB29) and red (probable cadmium red, PR108) Ben-Day dots are oilbased paints, which Lichtenstein applied by pushing the paint through a metal stencil with a toothbrush [2]. As has been noted with many modern oil paints [8], both passages exhibit mild water-sensitivity. The flat, blocked areas of bright yellow (lead chrome yellow, PY34), red (cadmium red, PR108) and black (Mars black, PBk11) areas are all thinly applied, coherent layers of Magna

\footnotetext{
${ }^{5}$ Roy Lichtenstein presented the original drawing to Tate in 1969. The sketch is now part of Tate's collection (graphite on paper, T01131). https://www.tate. org.uk/art/artworks/lichtenstein-drawing-for-whaam-t01131.

6 The documentary "Roy Lichtenstein", produced and directed by Chris Hunt in 1991, presents a detailed overview of Lichtenstein's artistic processes and development, based on a series of interviews with the artist. The documentary is available at this link: https://www.youtube.com/watch $? \mathrm{v}=\mathrm{FjJ} \mathrm{xcrjMlwU}$.
} 


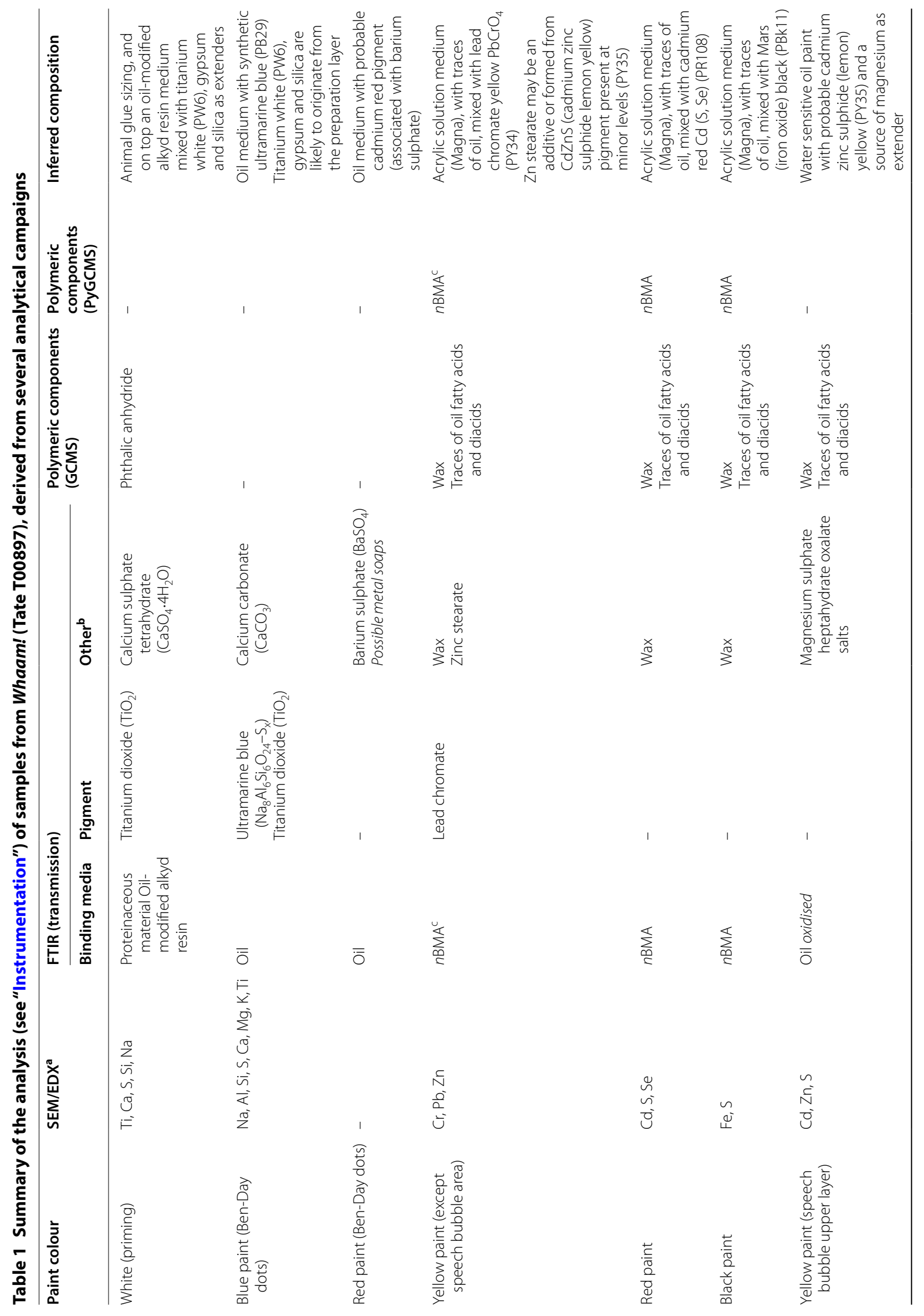




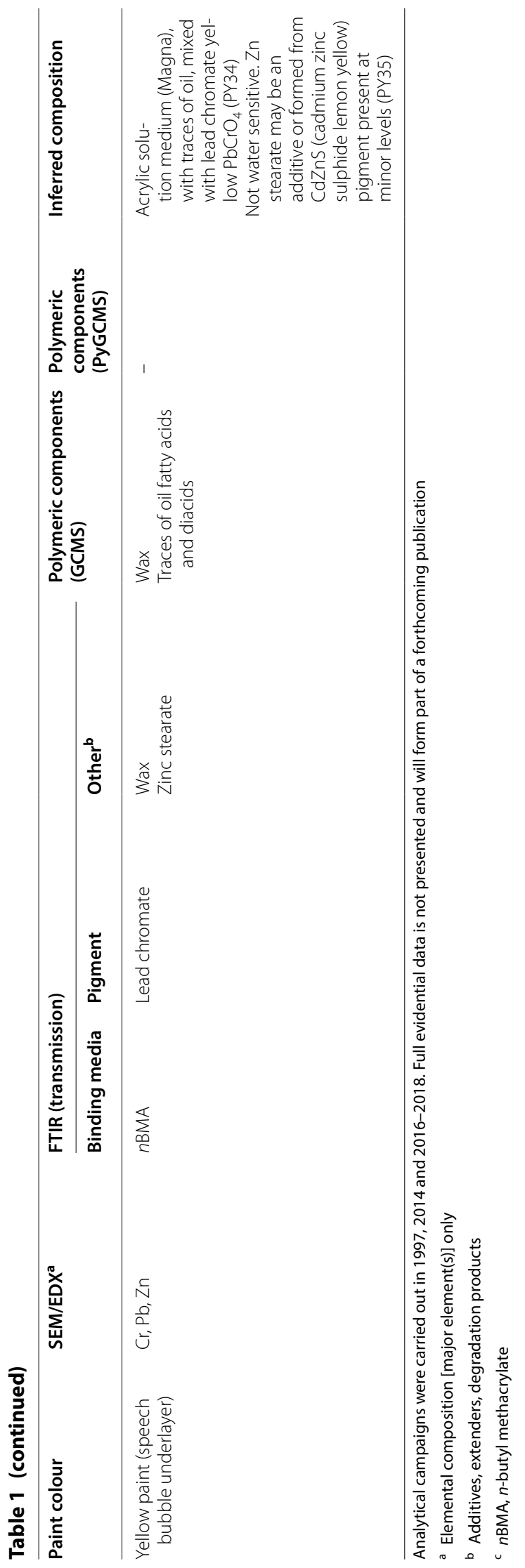


paint. Magna was developed in the late 1940s by Leonard Bocour and Sam Golden, consisting of an $n$-butyl methacrylate homopolymer ( $n \mathrm{BMA})$ dissolved in turpentine, mixed with pigment. Magna remains soluble in hydrocarbon-based solvents, and these passages have also been reported as being vulnerable to the prolonged action of water [7]. In addition to the block applications of Magna colour, Lichtenstein often reinforced his black lines with black Magna paint, where subtle brushwork and playful gloss shifts become evident upon closer inspection.

The analysis of small samples taken from the Magnabased areas in Whaam! revealed that these paints also contain wax and traces of oil fatty acids (Table 1). The presence of wax in Magna paint has already been noted by Upright [9], Gates et al. [10] and Rogge and Epley [11] and is thought to have been added to the original Magna formulation to provide body and stability. The presence of oil could be due to Lichtenstein adding oil medium to his Magna paints, or, as the levels detected were only minor, they may alternatively originate from the underlying alkyd priming. Lichtenstein stated that his preference for Magna paint over other media was related to its solubility in turpentine, which enabled him to make compositional changes without leaving traces of previous versions. He also discussed mixing Magna colours together, as well as mixing Magna paints with oil colours to achieve a desired hue and intensity [2]. However, Lichtenstein did not recall having done so for Whaam!. ${ }^{7}$

As an exception to the Magna-dominated yellow passages, the yellow paint in the speech bubble appears to be composed of two layers (Table 1). The uppermost layer contains oil paint with cadmium zinc yellow (PY35), often used for a lemon-yellow hue. Underlying this is another yellow layer dominated by the chrome yellow Magna paint (Table 1). This underlying layer presents a pinkish fluorescence under ultraviolet light, which appears similar to discreet areas at the surface of the speech bubble, which suggests that the speech bubble area consists of a heterogeneous blend of these two paints. Cross-section analysis however, did not reveal the presence of two distinct paint layers, which reinforces the suggestion that these two turpentine-diluted paints may

\footnotetext{
7 'Some of the earlier yellows I mixed green into to make it more lemony. I don't know if I was doing it at the time of Whaam! ... I don't mix the cadmium reds with anything-Magna has such a deep cadmium red medium. I mix the ultramarine blue with a little white. I mix the yellow with a little green. The yellow on Whaam! is probably just straight yellow Magna.' Crook J, Learner T. 2000, p. 119. [2].

${ }^{8}$ It is difficult, when examining a small number of paint samples, to definitively identify whether paints were initially blended on the palette, or whether blending occurred during painting. Nonetheless, in most cases, the yellow paints were dominated by oil (speech bubble) or Magna (all other locations); with some evidence for the other paint type also in most samples.
}

have blended at the painting surface. ${ }^{8}$ Interestingly, there is also little historical evidence for the manufacture of chrome yellow Magna paint [12], which is the subject of further investigation. ${ }^{9}$

\section{Whaam! paint and solvent sensitivity ${ }^{10}$}

Discreet swab tests (Additional file 1: Table S1) confirmed that aqueous-based systems proved optimal with respect to the removal of the deposited, yellowed soiling which was characterised as containing calcium carbonate, silica, gypsum and sodium and potassium salts, as well as trace amounts of drying oil components for the oil-containing passages. ${ }^{11}$ These tests also confirmed that the Magna acrylic and priming passages remained unaffected by deionised water until subjected to relatively long exposures (i.e. around 20 double rolls). ${ }^{12}$ Unsurprisingly, the uppermost speech bubble paint (Table 1), proved to be highly sensitive to water (Additional file 1: Table S1), rendering it particularly vulnerable to aqueous cleaning [13-15]. Microscopic examination (Fig. 3) revealed that the canvas weave structure is clearly visible through the thinly applied Magna (Fig. 3a) and priming layers (Fig. 3b). Equally, the moderately water-sensitive French ultramarine blue and cadmium red Ben-Day dots were characterised as thin inhomogeneous applications of oil paint (Fig. 3b); all of which underlined the need to minimise mechanical action across the entire painting surface.

\section{Methodology \\ Instrumentation \\ Digital microscopy}

High-resolution digital microscopy was performed on the painting surface and mock-up samples prior to cleaning, during and after cleaning trials, and after treatment using a Hirox KH-8700 microscope (Hirox, Japan) with an MXG-2500REZ revolver zoom lens set at 50 and $100 \times$ magnification, using ring light illumination. Images were processed using Hirox software.

\footnotetext{
${ }^{9}$ A more detailed characterisation of the paints found in Whaam! is the subject of an ongoing investigation, and to be published as a separate paper (under preparation)

${ }^{10}$ Prior to any surface evaluation, Whaam! was photographed under normal, raking, transmitted, infrared and ultraviolet lights, and the two canvases were brush-vacuumed both front and back.

11 The soiling layer was characterised via Fourier transform infrared spectroscopy (FTIR) and energy dispersive X-ray (EDX) analysis.

12 One swab roll is defined as a gentle forward and back motion over the surface. D5 silicone solvent was also tested on the alkyd priming, with poor soil removal; it was also tested on the speech bubble, where no pigment pickup or changes were observed, however, the mechanical action required excluded its use for this area.
} 

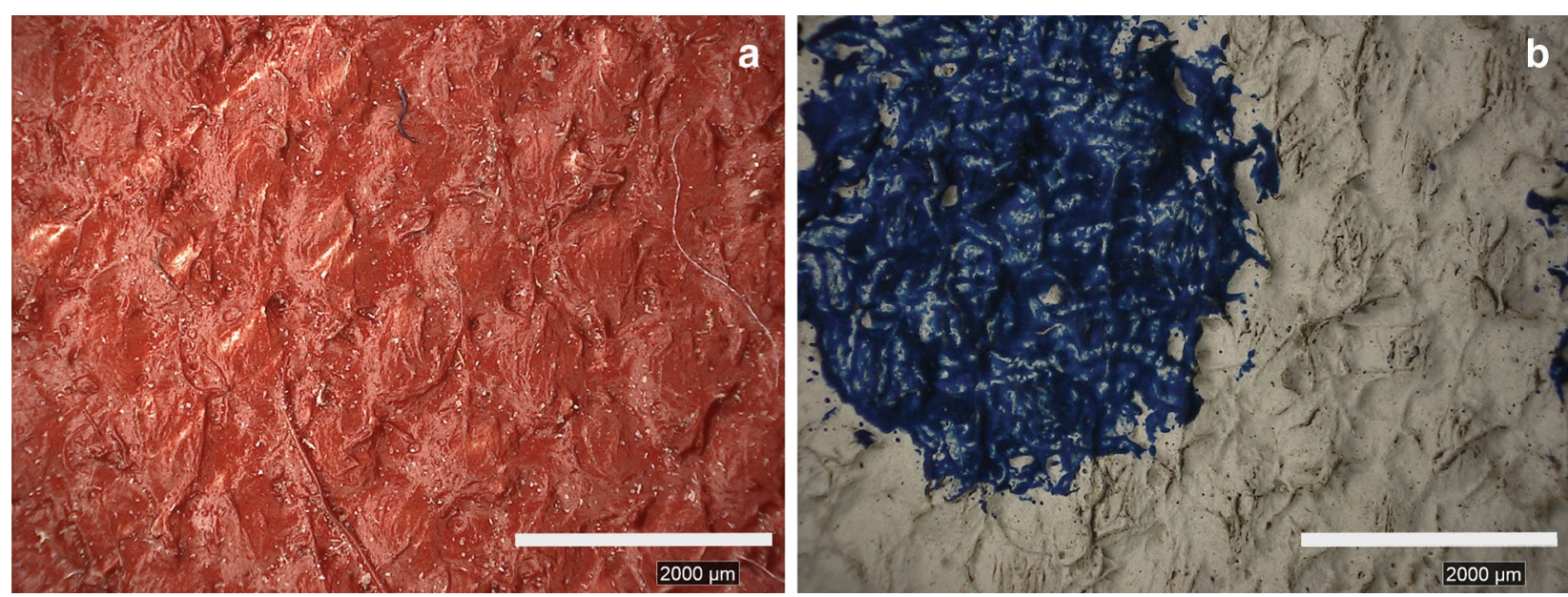

Fig. 3 Digital microscopy (Hirox) images showing the thin, well-bound applications of paint, as well as loose and ingrained dirt on the red Magna acrylic paint (a) and blue Ben-day dot and white alkyd priming (b) areas. The scale bar is $2000 \mu \mathrm{m}$, magnification is $\times 50$

\section{Colour measurement}

Measurement was performed on Whaam! prior to and after cleaning with a Konica Minolta CM-2600d spectrophotometer, using a D65 light source, a $10^{\circ}$ observer, collected in both specular components included (SCI) and excluded (SCE) modes. Measurement area was $\emptyset 8 \mathrm{~mm}$ and data were collected in the same locations as the gloss measurements (below), in triplicate. Values were averaged, and the standard deviation was calculated. The colour difference was determined using the CIE 2000 formula.

\section{Gloss measurement}

Measurement was performed on Whaam! prior to and after cleaning using a Rhopoint ${ }^{\mathrm{TM}}$ NOVO-GLOSS ${ }^{\mathrm{TM}}$ Trigloss glossmeter, collecting three incidence angles simultaneously $\left(20,60\right.$, and $\left.85^{\circ}\right)$. Measurement area was approximately $6 \times 2 \mathrm{~cm}^{2}$ with a resolution of 0.1 gloss units (GU). Final values were obtained using the $85^{\circ}$ angle based on assessment using the $60^{\circ}$ geometry where, if the gloss values at $60^{\circ}$ were higher than $70 \mathrm{GU}$, the $20^{\circ}$ value was used for the $\Delta$ gloss calculations; alternatively, if the value at $60^{\circ}$ was below $10 \mathrm{GU}$, the $85^{\circ}$ value was used.

\section{Energy-dispersive $x$-ray analysis (SEM/EDX)}

SEM/EDX elemental analysis was carried out on paint fragments and cross-sections taken from Whaam! and dried aqueous extracts from the painting surface using a LEO 1455VP scanning electron microscope (SEM) with INCA software, using back-scattered electron imaging (BSE), $20 \mathrm{kV}, 15-\mathrm{mm}$ working distance, and $100 \mathrm{~Pa}$ air pressure.

\section{FTIR spectroscopy}

Transmission FTIR spectroscopy was carried out using a Thermo Scientific Nicolet iN10 MX microscope, using a single diamond cell. 64 scans were collected at a resolution of $4 \mathrm{~cm}^{-1}$ across a 4000 to $600 \mathrm{~cm}^{-1}$ range, and data were processed using Omnic 9 software. Discreet water-droplet extracts were also performed on Whaam! to characterise the deposited soiling layer, via pipetting $50 \mu \mathrm{L}$ deionised water onto the paint surface (white alkyd, Blue Ben-Day dots, yellow, red and black Magna passages) for 5, 20 and $60 \mathrm{~s}$, as determined by individual paint responses. After extraction, the water droplets were pipetted onto glass slides and the dried extracts analysed in transmission mode.

ATR-FTIR (attenuated total reflectance) spectroscopy was carried out to explore cleaning system residues and changes to mock-up sample paint surfaces using a Thermo Scientific Nicolet iZ10 system and germanium ATR crystal. 64 scans were collected at a resolution of $4 \mathrm{~cm}^{-1}$ across a 4000 to $400 \mathrm{~cm}^{-1}$ range and data was processed using Omnic 9 software. Measurements were obtained in triplicate, and spectra were explored for characteristic absorption bands of the cleaning materials evaluated.

Micro reflectance FTIR-2D imaging was carried out on the mock-up samples to explore the presence of cleaning system residues using a Cary 620-670 FTIR microscope, equipped with a Focal Plane Array $128 \times 128$ detector (Agilent Technologies) in reflectance mode, with an open aperture and a spectral resolution of resolution of $8 \mathrm{~cm}^{-1}$ for 128 scans, which was selected to improve the signal-to-noise ratio of the reflectance spectra. The background was acquired using a gold plate. Control unsoiled 
and soiled areas, as well as areas treated with different cleaning systems, were analysed in triplicate. For each location, a map of $700 \times 700 \mu \mathrm{m}^{2}(128 \times 128$ pixels $)$ was produced, with a spatial resolution of $5.5 \mu \mathrm{m}$ (i.e. each pixel dimension is $5.5 \times 5.5 \mu^{2}$ ). For each two-dimensional (2D) map, the intensity of characteristic bands of cleaning solutions, emulsifiers and gels, was imaged and the chromatic scale of the maps shows increasing absorbance of the bands as follows (unless reported otherwise): blue $<$ green $<$ yellow $<$ red.

\section{Gas chromatography-mass spectrometry (GCMS)}

GCMS was carried out on very small paint samples removed from Whaam! to determine the drying oil components using a Varian Saturn CP-3900 GC coupled with a $1200 \mathrm{~L}$ single quadrupole detector. The oven program was set from 50 to $320{ }^{\circ} \mathrm{C}$ at $10{ }^{\circ} \mathrm{C} / \mathrm{min}$, then held for $5 \mathrm{~min}$ at $320{ }^{\circ} \mathrm{C}$ for a total run time of $33 \mathrm{~min}$. A splitless injection volume (injection port set at $300{ }^{\circ} \mathrm{C}$ ) of $1 \mu \mathrm{l}$ was used, with a helium flow of $1.0 \mathrm{~mL} / \mathrm{min}$. The column used was a Phenomenex Zebron ZB-5 column $(30 \mathrm{~m}$ length; $0.25 \mathrm{~mm}$ i.d.; $0.25 \mu \mathrm{m}$ film thickness). The mass spectrometer was used in EI mode $(70 \mathrm{eV})$; scan group 1: 45-300 amu; Group 2: 45-700 amu at $16 \mathrm{~min}$, every $1 \mathrm{~s}$. MS conditions: source temperature: $220{ }^{\circ} \mathrm{C}$; transfer line temperature: $270{ }^{\circ} \mathrm{C}$. Sample derivatisation was carried out using 3-(trifluoromethyl) phenyltrimethylammonium hydroxide $5 \% \mathrm{w} / \mathrm{v}$ in methanol by Alfa Aesar (Thermo Fischer Scientific). $12 \mu \mathrm{L}$ of derivatisation agent was added to a glass Reacti-vial $^{\mathrm{TM}}$ (Thermo Fischer Scientific), the vial was centrifuged, then heated to $60^{\circ} \mathrm{C}$ for $1 \mathrm{~h}$. The sample was centrifuged again, and $1 \mu \mathrm{L}$ was injected into the GC.

\section{Pyrolysis gas chromatography-mass spectrometry (PyGCMS)} PyGCMS analysis was carried out on the Magna paints using a CDS Pyroprobe 5000 heated Pt filament pyrolyser (CDS Analytical) and a Varian CP-3800 gas chromatograph coupled with a Varian Saturn 2000 mass spectrometer. Samples were injected in split mode (split ratio 1:50). The GC temperature was initially held at $50{ }^{\circ} \mathrm{C}$ for $2 \mathrm{~min}$, ramped at $10^{\circ} \mathrm{C} / \mathrm{min}$ to $310^{\circ} \mathrm{C}$, with a final hold of $10 \mathrm{~min}$. Total run time: $43 \mathrm{~min}$. Helium gas flow was set at $1.0 \mathrm{~mL} / \mathrm{min}$. Column: Phenomenex Zebron ZB-5 column (30 m length; $0.25 \mathrm{~mm}$ i.d.; $0.25 \mu \mathrm{m}$ film thickness). MS conditions: EI mode (70 eV); scanned 40-399 amu every $0.49 \mathrm{~s}$.

\section{Mock-up sample preparation}

The use of mock-ups to help facilitate research is well established. The process of making mock-ups offers insight into the artists' materials and techniques used, and the process of working with them helps conservators develop skills in the handling and optimisation of conservation treatment materials. For this study, four mockup samples were prepared with the aim of recreating Whaam! paint layers, using contemporary equivalent materials. A commercial cotton duck canvas was primed with Winsor and Newton (W\&N) titanium white alkyd [Griffin] paint, lightly diluted with Shellsol $\mathrm{T}$ solvent (Kremer Pigmente). French ultramarine blue W\&N Artists Oil Colour was used to create the blue Ben-Day dots, applied through a Polymex ${ }^{\circledR}$ stencil, mimicking Lichtenstein's technique of this period [2]. The other three primed canvases were painted with MSA (Mineral Spirit Acrylic Colors), a contemporary equivalent to Magna paint made by Golden Artist Colors, including cadmium yellow medium, ${ }^{13}$ cadmium red medium, and Mars black. After several days drying in ambient conditions, the samples were accelerated aged in a Sanyo MLH-351 environmental chamber, using Phillips daylight tubes with UV filters (average of 15,000 lx) at steady conditions of $25{ }^{\circ} \mathrm{C}$ and relative humidity of $55 \% \mathrm{RH}$. Mid-way through the ageing, an artificial soil mixture $[16,17]$, containing only the solid components (i.e. not suspended in solvent), was brush-applied to the mock-up surfaces, leaving an unsoiled area on each sample as a reference. The mockups were then further aged to enhance the bond between the soil and paint surfaces (see Additional file 1: Figure S1) to a total of 40 days, which approximates 25 years display in slightly elevated temperature museum conditions. A second set of naturally aged mock-ups, prepared in 2006 using azo yellow (PY3), burnt umber (PBr8) and titanium white (PW6) MSA paints (Golden Artist Colors, USA) on an oil-primed canvas, were also utilised for specific evaluations, and not artificially soiled to aide in the detection of cleaning system residues.

\section{Cleaning system selection}

Figure 4 represents the selection process for the solvents and application methods used, drawn from recent advances in the cleaning of modern painted surfaces such as the use of adjusted waters and a range of gels and emulsifiers, in addition to the novel materials introduced through the NANORESTART project. ${ }^{14}$ It was important to include this range of materials to demonstrate the advantages and disadvantages of each system, and to devise the most appropriate protocol for Whaam!.

\footnotetext{
${ }^{13}$ Although the majority of the yellow passages in Whaam! appear to contain chrome yellow pigment, no equivalent for chrome yellow Magna is available. Mark Golden is acknowledged and thanked for donating these paints to Tate.

14 NANORESTART was a 42-month collaborative research project (20152018) funded under the EU Framework Programme for Research and Innovation Horizon 2020 (Grant Agreement Number 646063), http://www. nanorestart.eu/.
} 


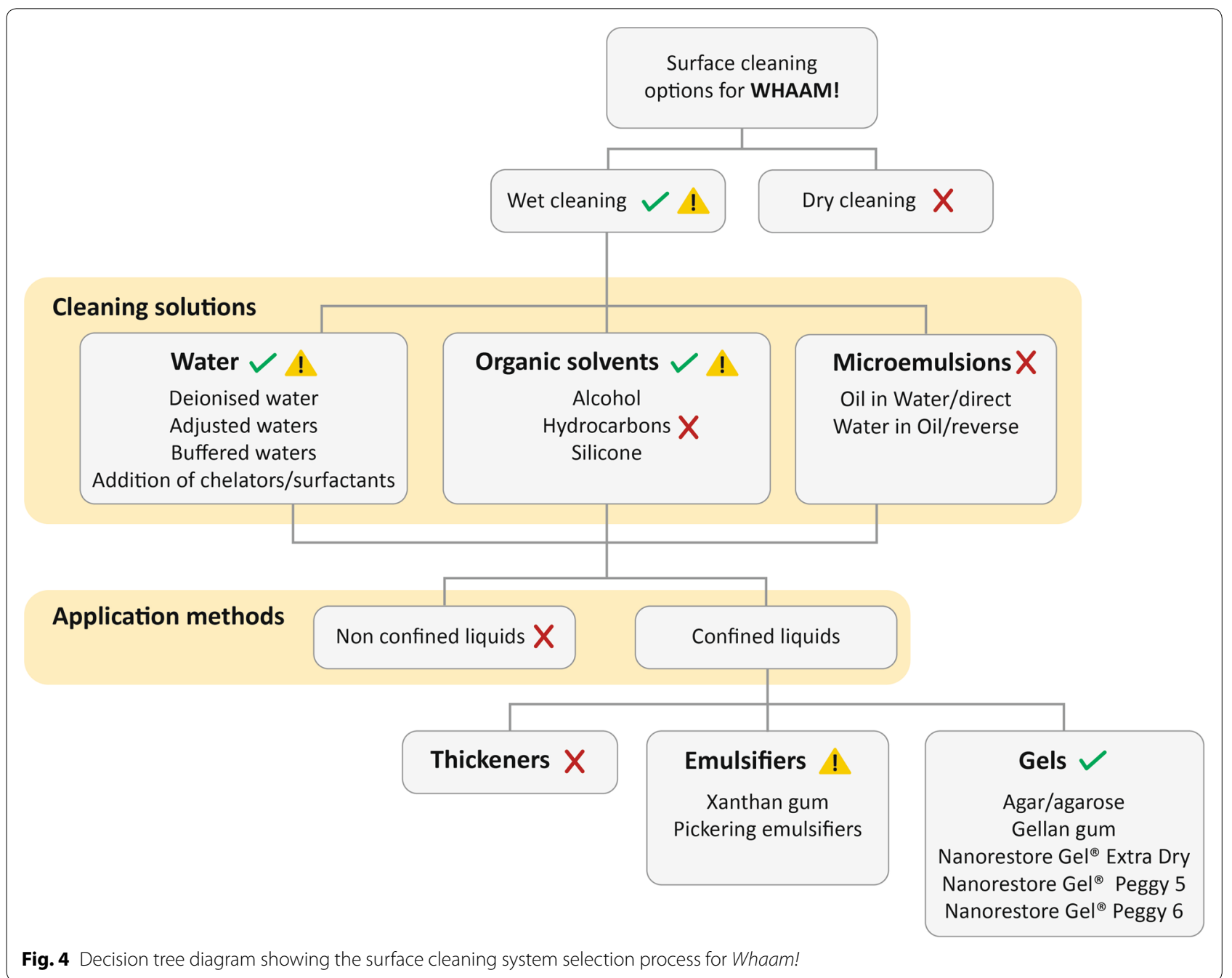

The requirements for a successful cleaning strategy included:

- the ability to employ the same materials and processes across the work in order to facilitate an even and consistent removal of the soiling layer across both panels;

- the ability to be tailored as required to each paint layer to account for subtle differences in paint texture, sensitivity and soiling adhesion;

- the ability to minimise mechanical action and an achieve an efficient cleaning action;

- not posing undue risk to any of the paint films through swelling, pigment pickup, gloss change and/ or cleaning system residues.

Due to the three paint types present, hydrocarbon solvents were ruled out due to the inherent of solubility of Magna paints in these solvents. Silicone solvents and aqueous options were initially trialled, where it was quickly determined that aqueous systems offered enhanced soiling removal. The additional ability to adjust aqueous $\mathrm{pH}$ and conductivity $[18,19]$ as well as adding chelators and/or surfactants to enhance cleaning efficacy was also considered advantageous (see Table 2). In addition, the cyclic silicone solvent decamethylcyclopentasiloxane (D5) did not unduly affect any of the paint surfaces, thereby offering potential as a masking layer or cleaning system base solvent (Fig. 4 and Table 2).

In order to reduce the risks associated with mechanical action, solvent penetration, pigment pickup and tideline formation, the free-liquids chosen would require confinement and application using a thickener/emulsifier/ gel. Of the possible candidates currently being used in conservation, spreadable aqueous-based emulsifiers such as xanthan gum $[20,21]$ were rejected due to the level of mechanical action required during cleaning and the need for an aqueous clearance step. Examples of Pickering 
silicone emulsifiers, such as Velvesil Plus, Shin-Etsu KSG 210 and Shin-Etsu KSG 350z ${ }^{15}$ [6, 21-24] were included (Fig. 4) due to the low impact of the silicone solvents on these paints, and the highly controlled aqueous exposures afforded by these systems. Aqueous-based rigid gels were also included, such as the polysaccharide gels Agarose and Gellan gum [25-28]. Another series of novel aqueous-based gels-the Nanorestore ${ }^{\circledR}$ series-which offer unique flexibility and solvent retention, and with the promise of enhanced performance were also considered. These gels form part of the range of materials developed by CSGI to avoid the limitations of traditional solvent thickeners [29]. Nanorestore Gel ${ }^{\circledR}$ Extra Dry (now MWR) ${ }^{16}$ is a transparent, rigid, chemical hydrogel consisting of a semi-interpenetrative network consisting of poly (2-hydroxyethyl methacrylate) and polyvinylpyrrolidone [30-35]. This gel offers useful liquid retention capacity which may be advantageous for surface-cleaning water-sensitive surfaces [36, 37]. The Nanorestore Gel ${ }^{\circledR}$ Peggy series ${ }^{17}[38,39]$ were specifically developed within the NANORESTART project for the cleaning of contemporary works of art with textured or irregular surfaces. These gels are opalescent, physical hydrogels based on a poly(vinyl alcohol) polymeric network. Nanorestore $\mathrm{Gel}^{\circledR}$ Peggy 5 consists of a blend of polyvinyl alcohol (PVA) and polyvinylpyrrolidone (PVP), where the PVP provides enhanced retention. Nanorestore Gel ${ }^{\circledR}$ Peggy 6 is made from PVA alone and is more flexible and elastic than Nanorestore Gel ${ }^{\circledR}$ Peggy 5, with the potential for enhanced conformation to moderately textured surfaces. Table 2 lists the preparation methods for each gel/emulsifier system taken forward to the mock-up samples.

\section{Cleaning system optimisation and evaluation methodology}

Designing the optimal cleaning strategy for Whaam! involved four phases, represented in Fig. 4.

1. Extensive evaluations using the free-liquid systems listed in Table 2 carried out on the mock-up samples and rated based on soiling removal efficacy and an evaluation of the cleaning test sites after clearing and drying (see Additional file 1: Table S2). The freeliquids were applied via hand-rolled cotton swabs rolled over the paint surface up to 10 times, with appropriate clearance steps applied where required,

\footnotetext{
${ }^{15}$ The Pickering silicone emulsifiers Shin-Etsu KSG350z (https://www.shine tsusilicone-global.com/products/personalcare/products/silicone_gels.shtml) can be bought at Kremer Pigmente, Germany https://www.kremer-pigmente. com/en/ready-made-colors/persist-solvent-gels/7616/ksg-350-z-silicone-gel.

16 http://www.csgi.unifi.it/products/dry.html.

17 http://www.csgi.unifi.it/products/peggy.html.
}

to cover an area of approximately $1 \mathrm{~cm}^{2}$. Trials were performed on both the unsoiled control areas and artificially soiled areas.

2. A selection of the most promising free-liquids was trialled discreetly on Whaam! applied as described above.

3. Confined systems (i.e. gels and emulsifiers) were evaluated on mock-up samples as prepared with deionised (DI) water only or combined with the most promising free-aqueous systems (Table 2). The cleaned areas were evaluated immediately and then again after the surface had dried, for characteristics such as: cleaning efficacy, ease of use (application, removal and clearance), relative conformation to the substrate surface and relative opacity (see Additional file 1: Table S3). The cleaned areas were also examined using digital microscopy to assess any impact on the paint surface and to explore the presence of cleaning system residues.

4. The final phase involved limited trials on Whaam! using a selection of optimised, confined systems in order to fine-tune the materials further, prior to commencing the conservation treatment.

\section{Cleaning system evaluation and star diagrams}

For the latter stages of the evaluation process, star diagrams were used to represent the results of the empirical evaluations, where the criteria (Additional file 1: Table S2) had been adjusted from previous research [40] to reflect the specific requirements of this case study. For each parameter of interest, each cleaning system was rated on a scale from 1 (inadequate/poor) to 5 (most appropriate), where larger stars represent more promising systems. The rating system was then further modified to capture parameters unique to the various gels and emulsifiers, as described in Additional file 1: Table S3.

\section{Results and discussion-part 1: cleaning system evaluation and optimisation}

Free-liquid trials-mock-ups

As expected, the neat cyclic silicone solvent D5 performed poorly with respect to cleaning efficacy [40, 41]. For the alkyd-based mock-up, no pigment pickup or gloss change was noted with this solvent, however slight pigment pickup was witnessed on the MSA (Magna equivalent) and oil-based Ben-Day dot mock-ups. D5 spreads on contact with paint surfaces and its slow evaporation rate delayed the visual assessment of treated surfaces. The hydrocarbon solvent Shellsol D40 also exhibited poor cleaning efficacy, and unsurprisingly caused pigment pickup on the MSA mock-ups and to some extent on the Ben-Day dot mock-ups (Additional file 1: Table S4). 


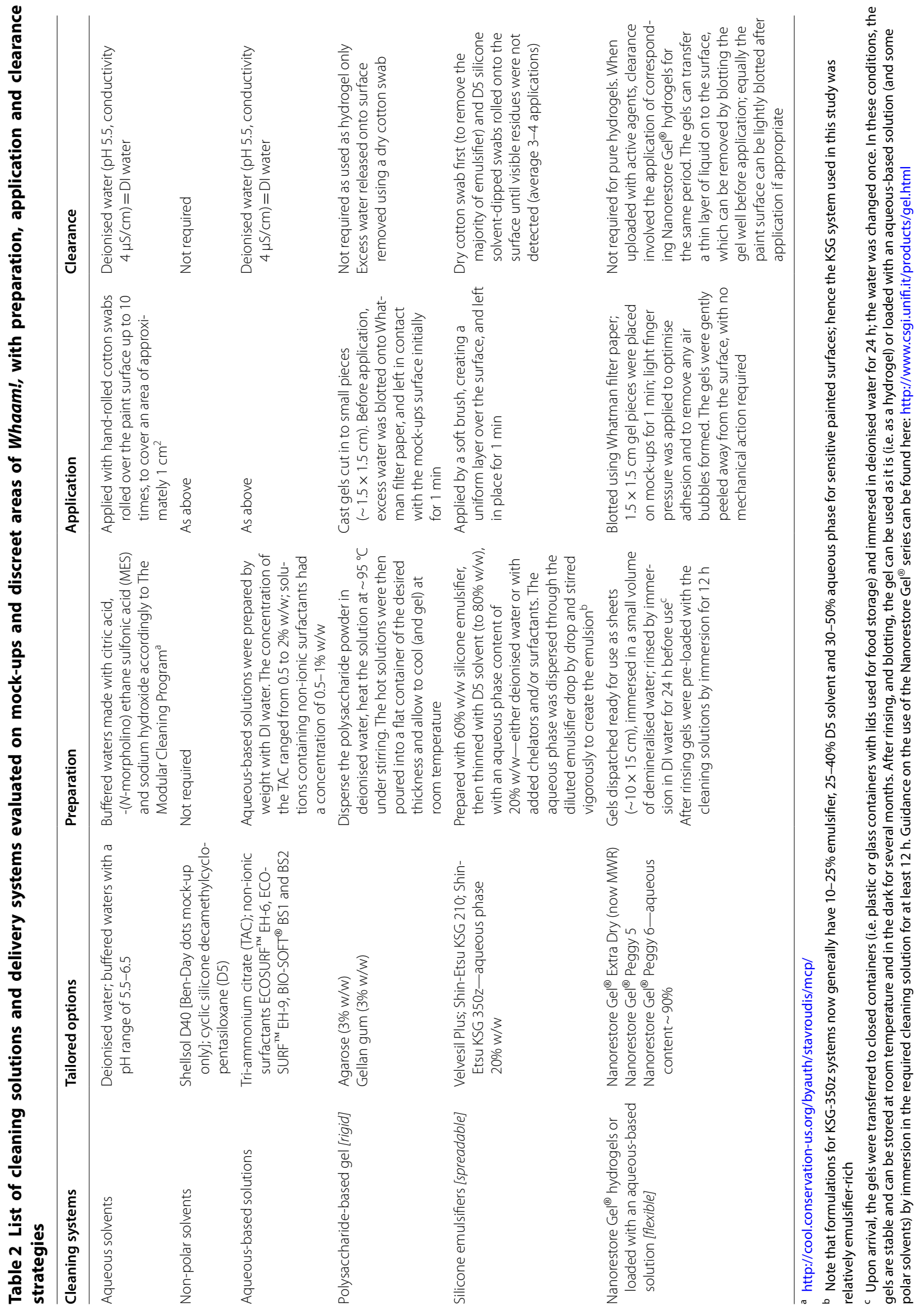


Deionised water exhibited an adequate cleaning efficacy and caused no detectable physical changes to both the alkyd and MSA-based mock-ups; whilst some pigment pickup was noted on the blue Ben-day dot mock-up.

The addition of tri-ammonium citrate (TAC) chelator to the DI water at a concentration of $0.5-1 \% \mathrm{w} / \mathrm{w}$, resulted in increased soiling removal efficacy to a moderate level for the MSA passages, and also performed well on the alkyd priming mock-up. Aqueous solutions with added non-ionic surfactants (see Table 2) also performed well, with improved cleaning efficacy noted with increased surfactant concentrations up to $1 \% \mathrm{w} / \mathrm{w}$. Whilst no visual changes were observed on the mockup surfaces after the use of aqueous surfactant solutions, foaming was noted for these systems on all MSA paints tested (Additional file 1: Table S4). As expected, foaming was more pronounced when concentrations were higher, confirming that lower quantities (e.g. $0.5 \% \mathrm{w} / \mathrm{w}$, up to $10 \times$ the critical micelle concentration $[\mathrm{CMC}])^{18}$ were optimal. Where performed, the deionised water clearance step often resulted in additional soil removal and did not produce significant alterations to any paint surfaces.

\section{Free-liquid selection-Whaam!}

Figure 5 summarises the results for preliminary tests carried out on discreet areas of the painting, ${ }^{19}$ with the most promising cleaning liquids, i.e. aqueous-based solutions with added TAC or non-ionic surfactant (see Table 2) at $\mathrm{pH}$ 5.5-6.5. None of the solutions tested caused discernible swelling or pigment pickup, however the Magna passages proved sensitive to mechanical action with prolonged swabbing action (i.e. more than 10 rolls). The $0.5 \% \mathrm{w} / \mathrm{w}$ TAC solution rated highly for the Magna paint passages with respect to soiling removal efficacy and paint surface integrity, however this solution proved less effective on the alkyd priming. The two non-ionic surfactant solutions did not perform as well as the TAC solution with respect to cleaning efficacy on both the alkyd priming and Magna paints, and, as was noted for the MSA mock-ups, foaming was also observed with the surfactant solutions-in this case most pronounced on the black Magna mock-up. Further tests using increased concentrations of up to $1 \% \mathrm{w} / \mathrm{w}$ surfactant and/or chelator (data not shown) resulted in enhanced soiling removal, nonetheless, any advantage was countered by the foaming observed for all surfactant-containing options.

Additional trials were also performed on Whaam! using pH buffered waters [19], as listed in Table 2. These

\footnotetext{
${ }^{18}$ The CMC of ECOSURF ${ }^{\circledR}$ EH-6 is 914 ppm at $25^{\circ} \mathrm{C}$; BIO-SOFT ${ }^{\circledR}$ BS2 is no longer available.

19 Due to the noted water sensitivity of the Ben-Day dot passages and the yellow speech bubble area, tests were restricted in these locations.
}

were created to evaluate the effect of $\mathrm{pH}$ on cleaning efficacy (MES buffered waters) and to compare the effects of citric acid-buffered waters to TAC solutions. Although moderate soil removal was noted using the range of buffered waters, aqueous solutions with added TAC consistently produced optimal results across the painting surface with respect to soiling removal efficacy and the retention of paint surface character. Figure 6 shows the corresponding star diagram for the alkyd priming evaluations, where the $0.5 \% \mathrm{w} / \mathrm{w}$ TAC solution (at $\mathrm{pH} \sim 6.5$ ) performed similarly to the MES water buffered to $\mathrm{pH} 6.5$; however, in other areas of the painting, the TAC solution offered a more consistent soiling removal action, which was key to the success of this treatment.

\section{Gel and emulsifier selection-hydrogel evaluations- mock-ups}

During this phase, the range of confining materials, i.e. gels and emulsifiers (Table 2) were tested as prepared with deionised water only to compare their ease of use, inherent cleaning efficacy and physical properties such as transparency/opacity and relative conformation to the various paint surfaces. The results are presented for the black MSA mock-up (Figs. 7 and 8) and the oil Ben-Day dot mock-up (Fig. 9). The black MSA mock-up proved particularly useful due to the relative visibility of the cleaning tests, which informs treatment options for these relatively under-studied paints [27].

The agarose and gellan gum options offered minimal cleaning efficacy after a 1-min exposure and a second application using a 5-min exposure produced similar results. No swelling, blanching, pigment pickup or gloss change was observed on any of the mock-ups using these gels. Their application and removal was straightforward, though it was noted the gels could break apart when handled. Surface contact was also not optimal and contributed to uneven soil removal and the use of weights was not desirable for Whaam! After the removal of the gels, small water droplets were also observed on the mock-up surfaces, which required removal using a dry cotton swab. Due to these issues, the two rigid polysaccharide gels were not taken forward for further modification and evaluation.

For the Pickering silicone emulsifiers (Table 2), promising results were obtained with respect to soil removal with the KSG 350z option (see Figs. 7 and 9). However, the low evaporation rate of the clearance solvent (D5) ${ }^{20}$ made it difficult to assess paint surfaces within a suitable period. It

\footnotetext{
${ }^{20}$ The use of silicone solvents, such as octamethylcyclotetrasiloxane (D4) and decamethylcyclopentasiloxane (D5), has been restricted by the European Commission in rinse-off cosmetic products with a concentration of $0.1 \%$ or more of either substance, due to their toxicity potential and because they tend to accumulate in the environment with unpredictable long-term effects.
} 


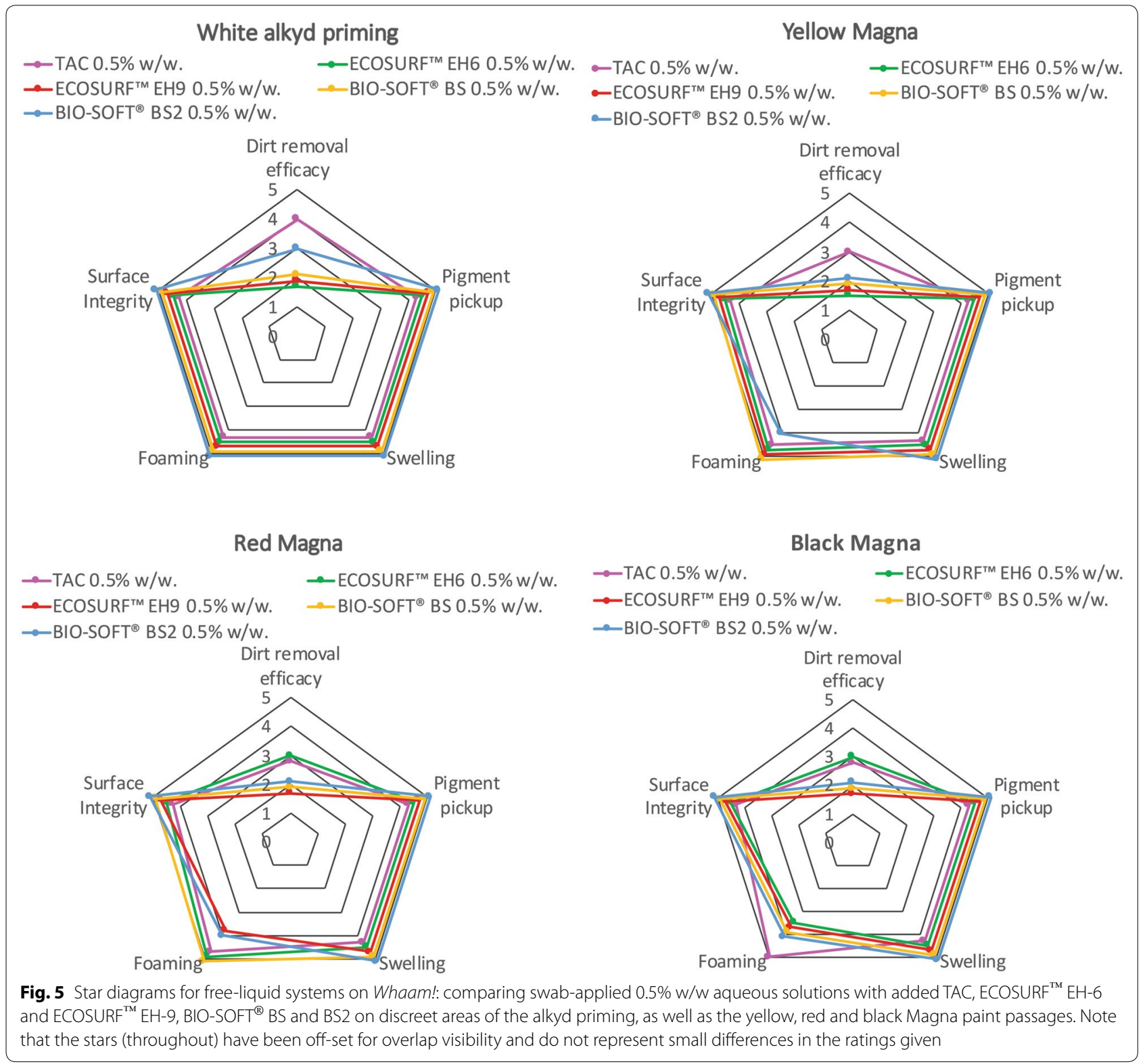

was also observed that the removal and clearance procedure is relatively time-consuming. Initially, clearance procedures were performed using a cotton swab dipped into D5 solvent, which was lightly dried off onto a paper towel and then rolled onto the mock-up surface. In this case, the emulsifier could be spread across the paint surface, making removal more difficult. The procedure was then modified by removing the bulk of the emulsifier with a dry swab, followed by a series of D5-dipped hand-rolled swab applications (average of 4). This extended clearance procedure resulted in paint softening, with consequent pigment pickup and surface changes noted for the MSA and Ben-Day dot mock-ups, as also noted during the free-liquid trials. As a result, the emulsifiers received a low rating for surface integrity and pigment pickup (Figs. 7 and 9), in addition to health and safety concerns around the risks associated with silicone materials. ${ }^{21}$

Nanorestore Gel ${ }^{\circledR}$ Extra Dry (now MWR) is a rigid gel which was easy to apply and remove from paint surfaces. The gel did however partially release water onto mock-up surfaces, which was removed via blotting using a Whatman filter paper. This hydrogel resulted in a moderate cleaning efficacy (see Figs. 7 and 9), which represented an enhanced performance when compared to agarose and gellan. No residues

\footnotetext{
${ }^{21}$ https://www.chemsafetypro.com/Topics/Restriction/Restriction_of_D4_ and_D5_in_Personal_Care_Products.html.
} 


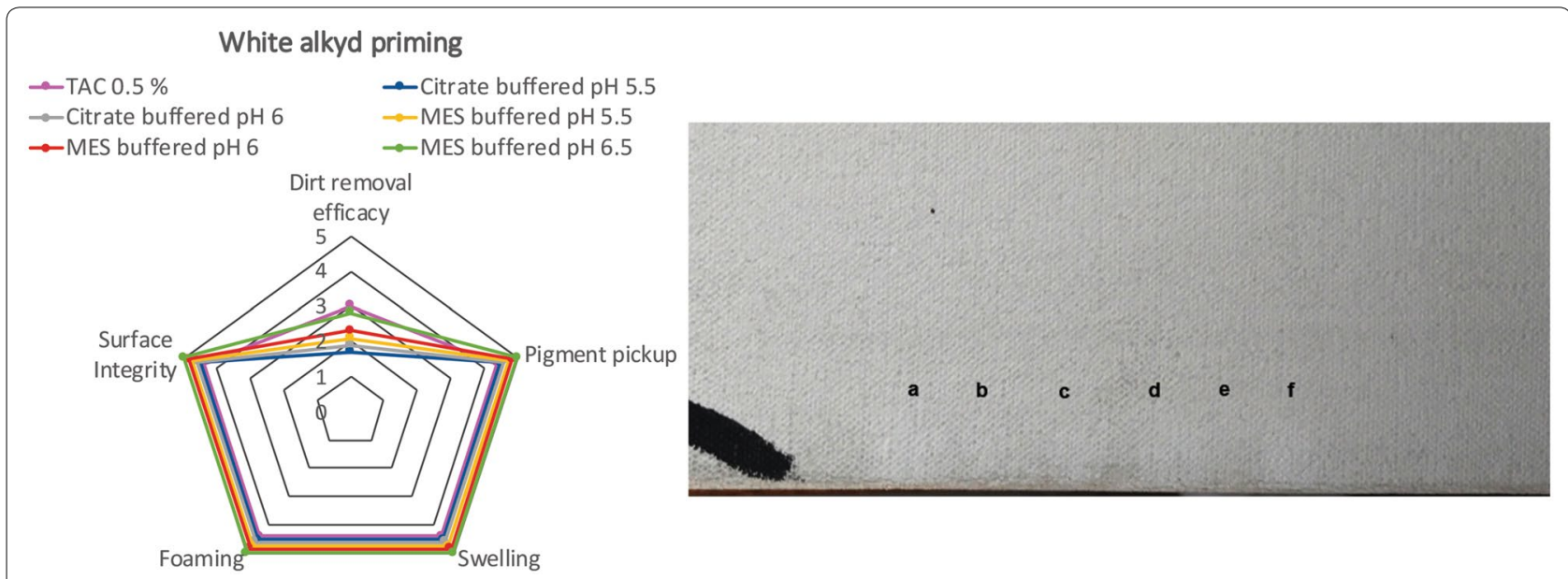

Fig. 6 Star diagram for Whaam! (on the left) — comparison between swab-applied buffered waters and aqueous solution with TAC at a concentration of $0.5 \% \mathrm{w} / \mathrm{w}$, for discreet areas of the alkyd priming. The image shows cleaning tests with $a \mathrm{TAC} 0.5 \% \mathrm{w} / \mathrm{w} ; b$ citrate buffered water $\mathrm{pH} 5.5$; c citrate buffered water $\mathrm{pH}$ 6; $d$ MES buffered water $\mathrm{pH}$ 5.5; e MES buffered water $\mathrm{pH}$ 6; and $f$ MES buffered water $\mathrm{pH} 6.5$

were noted, and the paint surfaces did not appear to be affected when examined under magnification (Figs. 8 and 9).

Both Nanorestore Gel ${ }^{\circledR}$ Peggy 5 and Nanorestore Gel ${ }^{\circledR}$ Peggy 6 performed optimally regarding soil removal, ease of use, and health and safety. They also conformed more fully to the paint and canvas textures than any of the other non-spreadable gels due to their unique flexibility; where contact with the paint surface could be further improved using light finger pressure. Their application and removal required a gentle placing onto, and then peeling away of the gels from the paint surface. Microscopic examination revealed that the artificial soil layer had been substantially reduced (see Figs. 8 and 9); however, as remnants of the soil remained, longer exposure times and the addition of chelators and/or surfactants to the aqueous phase would enhance cleaning efficacy. The presence of residual soiling was naturally more evident on lighter samples, such as the alkyd priming mock-up (see Fig. 9) and the yellow MSA samples (see Additional file 1: Figure S2). No gel residues were observed for any of the Nanorestore Gels via microscopy (Figs. 8 and 9; Additional file 1: Figure S2), however it was noted that the gels were tacky enough to pick up fibres from the blotting paper (Whatman filter paper) used to dry off the gels prior to use. It was also noted that these gels can deposit a very thin layer of water onto the paint surface, which can be reduced by blotting the gels more thoroughly prior to application and/or very lightly blotting the painting surface immediately after the removal of the cleaning and/or clearance gels.

In summary, for the deionised water-prepared range of gels and emulsifiers, the Nanorestore $\mathrm{Gel}^{\circledR}$ products Peggy 5 and Peggy 6 proved to be the most suited to the mock-up samples. They offered a moderately high cleaning efficacy with the advantages of minimal mechanical action, no pigment pickup and the ability to judge the surface soon after application and clearance. Hence, these gels were taken forward to the next evaluation phase, with the Velvesil Plus silicone emulsifier also included as an initial comparison. In retrospect, the KSG-350z emulsifier would have been a more helpful choice due to its enhanced modifiability over Velvesil Plus [6, 21, 24].

\section{Optimising gels and emulsifiers-mock-ups}

The initial series of gel/emulsifier evaluations confirmed that even when using deionised water alone, the rigid gels and emulsifiers offered significant advantages over the application of the free-liquid options with respect to cleaning efficacy; minimising unwanted changes to paint surfaces and reducing mechanical action. Trials were therefore expanded to include optimised aqueous solutions (see Fig. 6). Further investigations were carried out into different tissues for blotting the gels before application which determined that non-cellulosic, non-woven Evolon tissue ${ }^{22}$ proved helpful for drying the gels prior to use, with no fibre transfer and a comparable dry absorption capacity to Whatman paper.

Figure 10 contains an annotated diagram of the optimised aqueous gel tests performed on the black MSA mock-up, with corresponding star diagrams. Although the addition of chelators and/or surfactants improved the cleaning efficacy, it was noted that the relatively long exposures and clearance steps required for the silicone

\footnotetext{
22 https://www.preservationequipment.com/Catalogue/Conservation-Mater ials/Materials-and-Fabrics/Evolon-Microfilament-Material and https://www. talasonline.com/Evolon.
} 


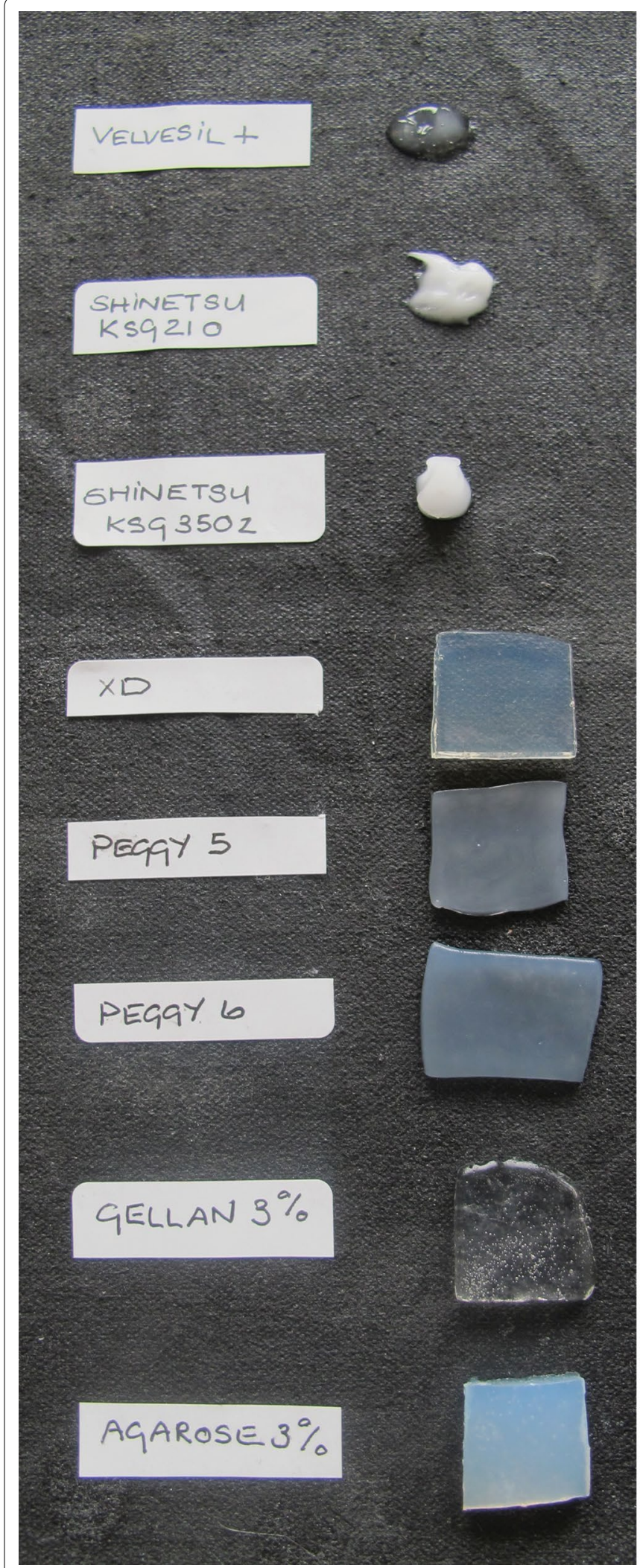

$\rightarrow$ Velvesil Plus 20\% w/w. aqueous phase

$\rightarrow$-Shin-Etsu KSG 210 20\% w/w. aqueous phase

$\rightarrow$-Shin-Etsu KSG 350z 20\% w/w. aqueous phase

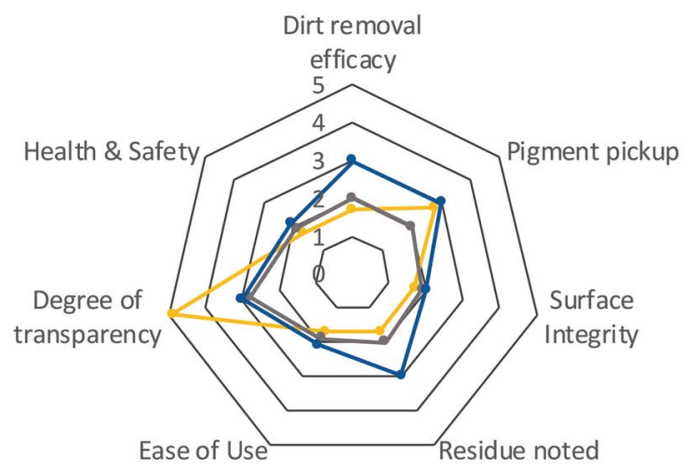

-Nanorestore Gel ${ }^{\circledR}$ Extra Dry

$\rightarrow$ Nanorestore Gel ${ }^{\circledR}$ Peggy 5

$\rightarrow$ Nanorestore Gel ${ }^{\circledR}$ Peggy 6

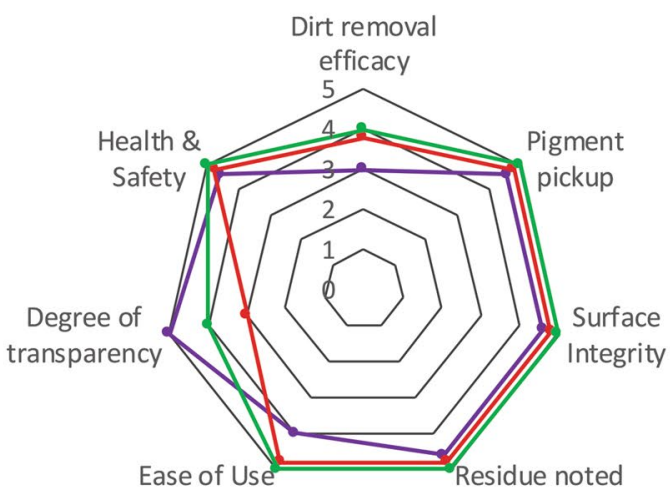

-Agarose $3 \% \mathrm{w} / \mathrm{w}$.

- Gellan gum 3\% w/w.

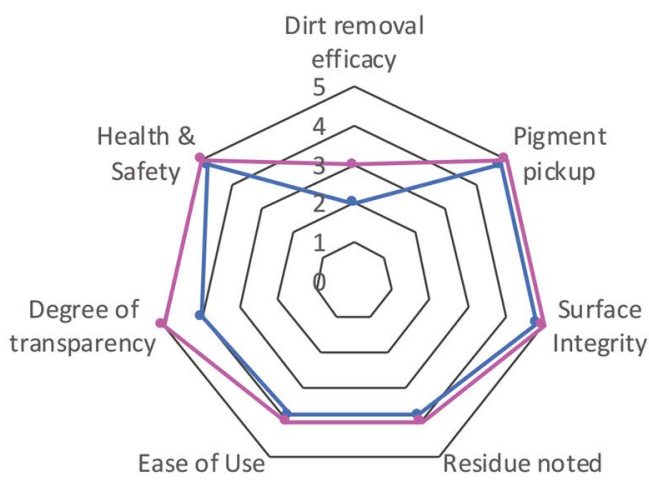

Fig. 7 The gel and emulsifier systems evaluated on the soiled black MSA mock-up and corresponding grouped star diagrams showing the performance of each as prepared with deionised water only. Note that the 'degree of transparency' was judged during use 


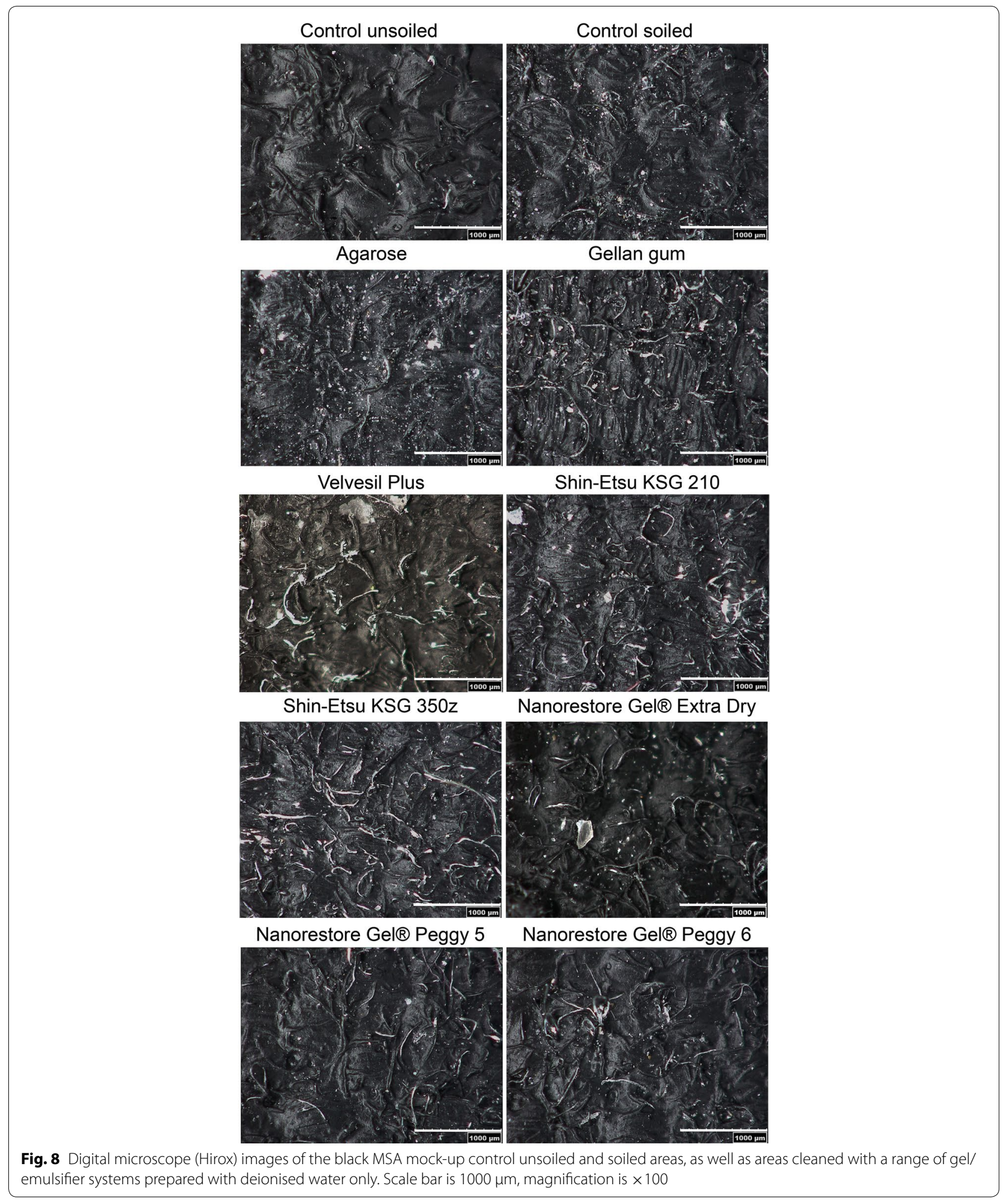

emulsifier resulted in softening of the paint and pigment pickup in some cases (e.g. for the oil Ben-Day dots); hence these materials were excluded from further trials.
As reflected in Fig. 10, among the Nanorestore $\mathrm{Gel}^{\circledR}$ products evaluated, the Peggy 5 and Peggy 6 gels proved optimal. Their unique flexibility and enhanced 


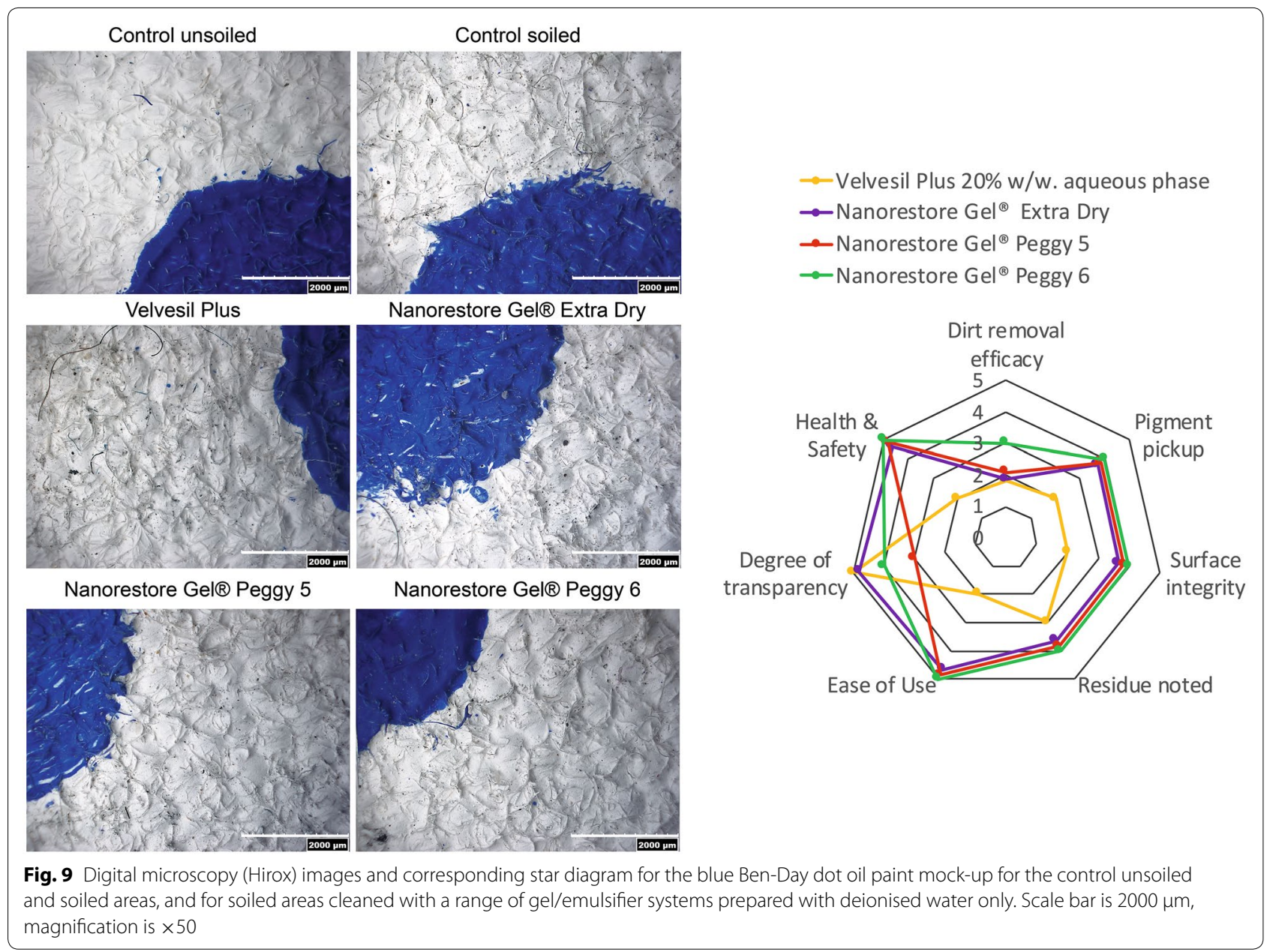

conformation to the substrate resulted in the most homogeneous removal of the soiling layer when compared to the Nanorestore Gel ${ }^{\circledR}$ Extra Dry (now MWR) and other gels/emulsifiers evaluated.

Similar cleaning efficacy results were obtained with both Peggy gels loaded with enhanced aqueous cleaning solutions, across the range of mock-ups. The gels loaded with TAC at $0.5 \% \mathrm{w} / \mathrm{w}$ consistently proved to be the most suitable, receiving high scores for all empirical criteria. For the tests where non-ionic surfactants (initial concentration $1 \% \mathrm{w} / \mathrm{w}^{23}$ ) were added to the aqueous phase, it was noted that the Peggy gels tended to become slippery and difficult to handle, with a loss of surface contact. As a result, a low score was allocated for the "ease of

\footnotetext{
${ }^{23}$ Preliminary tests on Whaam! using swab-applied cleaning solutions showed that, for concentrations higher than $0.5 \%$, foaming was observed at the paint surface for all the surfactants tested (particularly for the MSA paints). For use in gels, the concentration of the surfactant was increased to $1 \% \mathrm{w} / \mathrm{w}$, which proved to be more effective for soiling removal, where any foaming was minimised by confining the surfactant.
}

use" parameter, and observations of changes to the paint surface (e.g. slightly patchy, matte paint after both the cleaning and clearance steps) is also reflected in Fig. 10. Additional tests were performed with reduced concentrations of the surfactants, where it was observed that for concentrations equal or higher than $0.75 \% \mathrm{w} / \mathrm{w}$, the gels became difficult to handle and resulted in reduced contact with the substrate.

Figure 11 includes images of the black MSA mock-up cleaned with the Peggy 5 and Peggy 6 gels respectively. Both gels offer a similar cleaning efficacy, however as none of the options entirely removed the soiling layer, higher concentrations of surfactant and/or chelators, or longer exposure-times, or both, were considered. However, for the reasons outlined above, the use of surfactants in these gels was not taken forward. Although similar results were obtained using both Nanorestore Gel ${ }^{\circledR}$ Peggy gels; Peggy 6 was selected for preliminary testing on Whaam! as it is slightly more flexible, offers enhanced conformation to the surface, and as it is less 


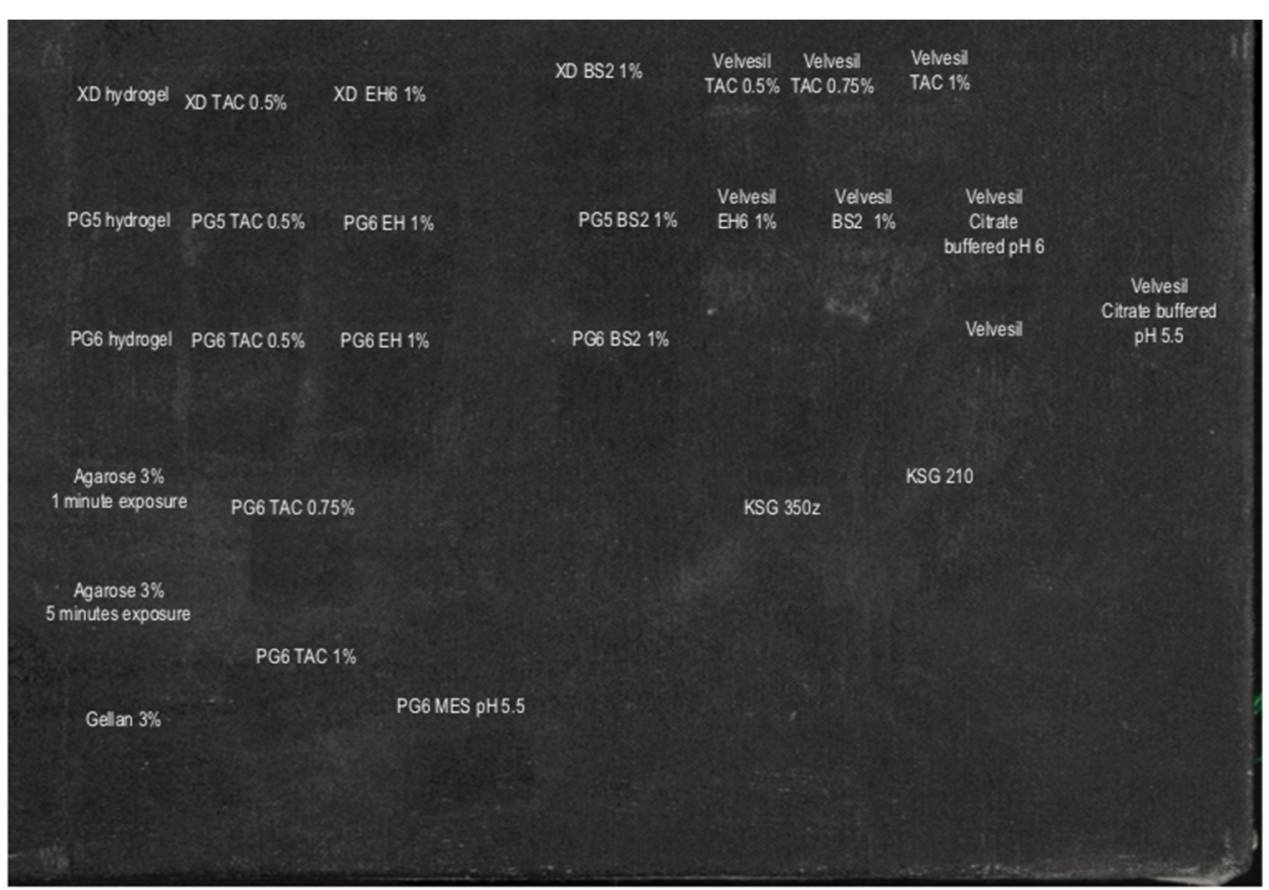

Velvesil Plus $20 \%$ w/w. aqueous phase

Nanorestore Gel` Extra Dry

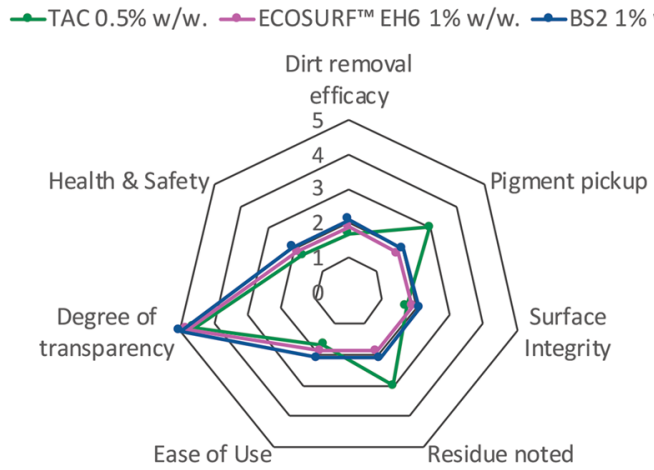

Nanorestore Gel` Peggy 5

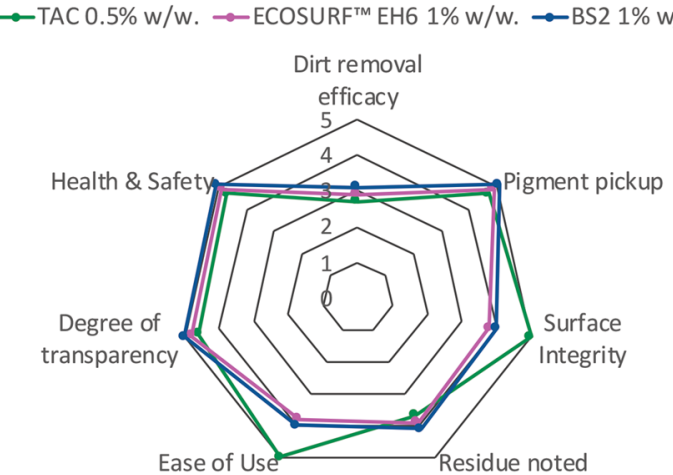

Nanorestore Gel` Peggy 6
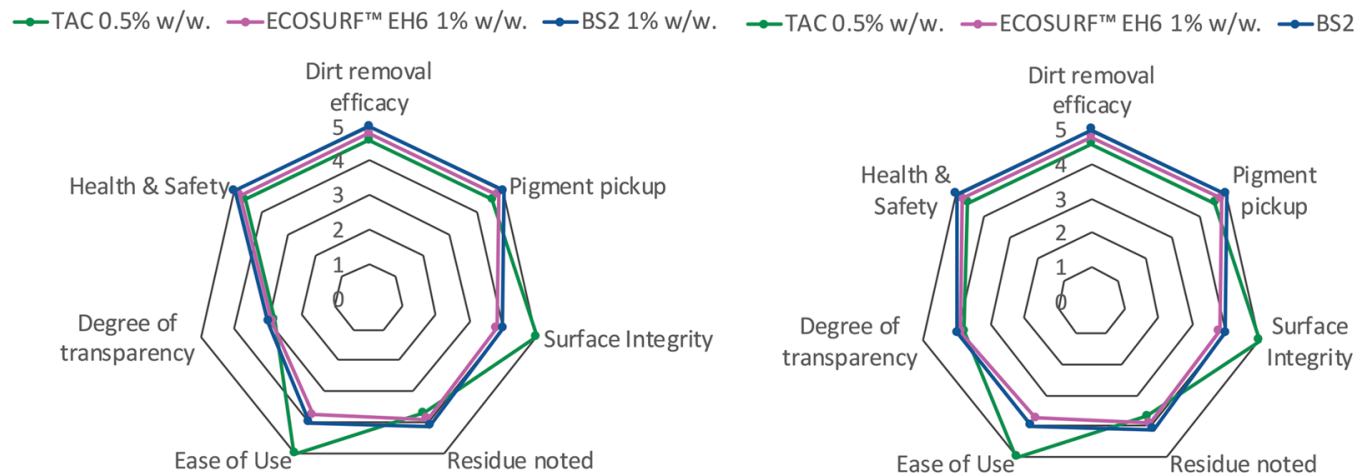

Fig. 10 Optimising cleaning tests performed on the soiled black MSA mock-up (top left) and corresponding star diagrams documenting various Nanorestore ${ }^{\circledR}$ gels and Velvesil Plus silicone emulsifier with 20\% aqueous phase. N.B. Nanorestore Gel ${ }^{\circledR}$ products received a score of 4 for the "residue noted" because they transferred a very thin layer of aqueous phase onto the surface (and subsequently blotted away as desired) 
opaque, it also offered enhanced visual access to the paint surface during application.

\section{Cleaning system residue evaluation-mock-ups}

The black MSA mock-up was also used to investigate the possible presence of cleaning system residues (gel, chelator, etc.) remaining on paint surfaces after clearance, which forms a necessary part of the evaluation of any novel cleaning materials. Alongside visual observations and microscopy (Figs. 7, 8, 9, 10, 11, and Additional file 1: Figure S2), two infrared spectroscopy techniques; ATRFTIR and micro-reflectance 2D-imaging were employed (see "Instrumentation").

For the range of gels and emulsifiers evaluated (Table 2), no residues were detected on the mock-ups using the ATR-FTIR system. Analysis of a select group of cleaned samples using microFTIR-2D imaging proved to be more sensitive, offering a lower detection limit of $\sim 5 \mathrm{mg} / \mathrm{m}^{2}$. The presence of possible residues on the black mock-up surface were explored via mapping characteristic absorption bands from each cleaning material: for example, the silicon-methyl stretching band at $1260 \mathrm{~cm}^{-1}$ for the silicone emulsifier, and the $\mathrm{C}-\mathrm{O}$ stretching band for polyvinyl alcohol at $1251 \mathrm{~cm}^{-1}$ for the Nanorestore ${ }^{\circledR}$ Peggy 6 gel. As noted during the ATR-FTIR analysis, the presence of absorption bands from the artificial soiling components dominated the acquired spectra. However, it was still possible to detect residues on areas cleaned with the silicone emulsifiers Shin-Etsu KSG 210 and Shin-Etsu KSG 350z, as shown in Fig. 12, though it is also noted that the proportion of emulsifier used was relatively high. The presence of silicone emulsifier residues has however been reported elsewhere, including when sponges were used (instead of swabs) for the clearance step [40]. Under the same analysis conditions, no residues were detected for any of the Nanorestore Gel ${ }^{\circledR}$ Peggy 5 and Peggy 6 gel options, shown in Figs. 13 and 14 respectively.

The Nanorestore Gel ${ }^{\circledR}$ Peggy 6 system was further investigated via simulating the cleaning procedure on a series of unsoiled MSA paints [Golden Artist Colors], see "Instrumentation". For these samples, key evaluations were carried out using the Nanorestore $\mathrm{Gel}^{\circledR}$ Peggy 6 loaded with deionised water and with an aqueous solution containing $1 \% \mathrm{TAC} \mathrm{w} / \mathrm{w}$, with exposure times of 2 and 15 min respectively, cleared via applying a hydrogel for the same period. Peggy 6 gel residues were investigated by mapping the polyvinyl alcohol $\mathrm{C}-\mathrm{O}$ stretching band at $1251 \mathrm{~cm}^{-1}$, whilst TAC residues were explored through mapping the symmetric oscillation band of the carboxylate ion at $1550 \mathrm{~cm}^{-1}$. Representative FTIR-2D imaging maps for the Azo Yellow MSA paint sample are shown in Figs. 15 and 16. In summary, there were no detectable residues on any of the unsoiled MSA mock-ups ascribable to the Peggy 6 gels and TAC solutions, confirming that any residues remaining on these paint films fall below the $5 \mathrm{mg} / \mathrm{m}^{2}$ instrument detection limit.

\section{Results and discussion-part 2: painting treatment Optimising the cleaning strategy-Whaam!}

After completing the cleaning evaluations and residue studies on the range of mock-up samples, a series of 1-min exposure tests were carried out on discreet areas of Whaam! using small $(1 \times 1 \mathrm{~cm})$ pieces of Nanorestore Gel $^{\circledR}$ Peggy 6 loaded with TAC at $0.5 \% \mathrm{w} / \mathrm{w}$, followed by a clearance step using a Peggy 6 hydrogel (deionised water) for the same period. Trials demonstrated that the three paint types required different concentrations of the TAC solution, and/or longer exposure times to achieve an even level of soiling removal. Further tests were carried out with varied TAC concentrations $(0.5-1 \% \mathrm{w} / \mathrm{w})$ and increasing the exposure time by $30 \mathrm{~s}$ intervals, up to $2 \mathrm{~min}$, followed by a similarly-timed clearance step. Trials were also performed on the blue Ben-Day dot and yellow Magna passages, however the changes-though visible to the naked eye-were too subtle to be captured using microscopy.

As shown in Fig. 17, for the red and black Magna passages, the TAC solutions at $0.75 \%$ and $1 \% \mathrm{w} / \mathrm{w}$ were found to be most effective at removing the soiling layer. However, at the $1 \% \mathrm{w} / \mathrm{w}$ concentration, very slight changes in surface gloss were observed under magnification, suggesting a subtle disturbance to the uppermost paint surface. In addition, when the used 1\% TAC gels were closely examined, faint pigment pick-up was noted. As a result, the lowest effective TAC concentration was selected for the Magna areas. The alkyd priming areas proved more tenacious, with higher quantities of soiling retained within this relatively textured layer. For these areas, the lower TAC concentrations only partially removed the soiling layer, hence the concentration was increased incrementally up to a maximum of $2 \% \mathrm{w} / \mathrm{w}$. Here the Nanorestore Gel ${ }^{\circledR}$ Peggy 6 gel loaded with TAC at $2 \% \mathrm{w} / \mathrm{w}$, at a 2 -min exposure (followed by the appropriate clearance step), was found to be more effective than repeated exposures for shorter periods using gels with lower TAC concentrations (Fig. 18).

Trials on the Ben-Day dot areas confirmed that optimal soiling removal and an even cleaning action was achievable using the Nanorestore Gel ${ }^{\circledR}$ Peggy 6 and TAC solution at $0.75 \% \mathrm{w} / \mathrm{w}$. There was no evidence of pigment pickup for the sensitive French ultramarine blue and cadmium red oil paints noted until the TAC concentration reached $1-1.5 \% \mathrm{w} / \mathrm{w}$; which could 
Nanorestore Gelß Peggy 5 TAC $0.5 \% \mathrm{w} / \mathrm{w}$.

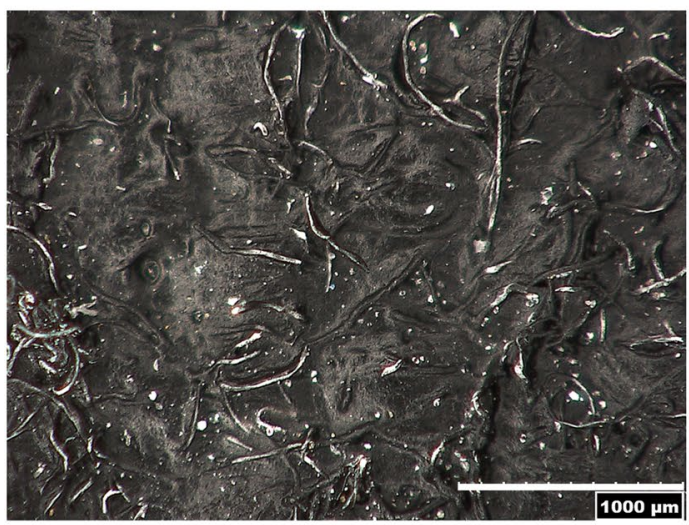

Nanorestore Gelß Peggy 5 EH6 1\% w/w.

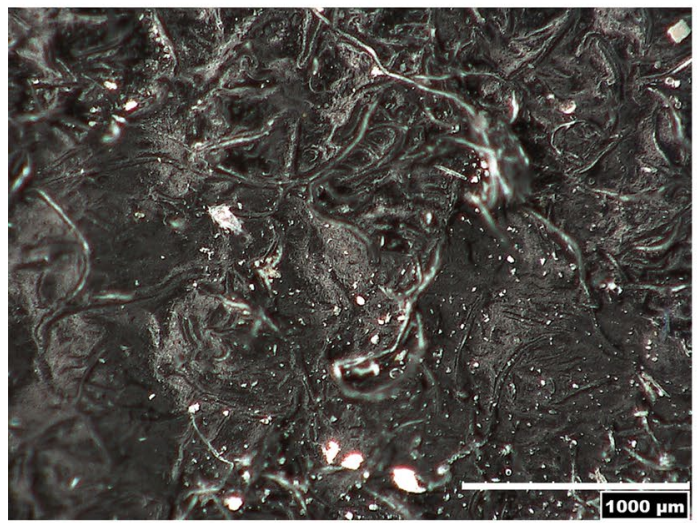

Nanorestore Gel囚 Peggy 5

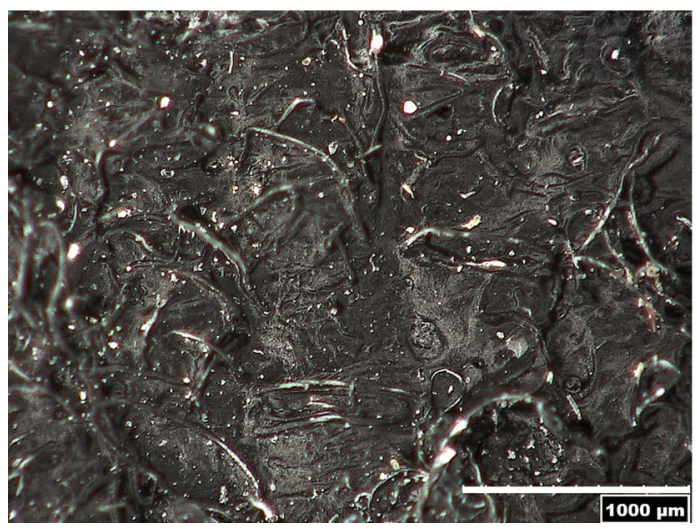

BS2 $1 \% \mathrm{w} / \mathrm{w}$.

Nanorestore Gelß Peggy 6

TAC $0.5 \% \mathrm{w} / \mathrm{w}$.

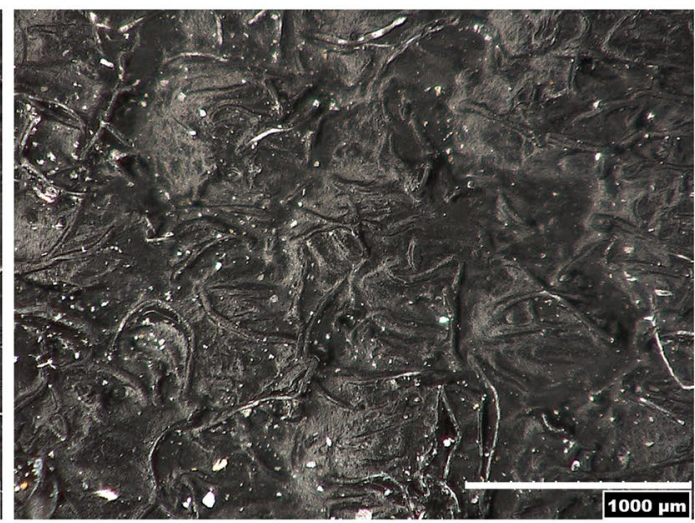

Nanorestore Gel® Peggy 6 EH6 1\% w/w.

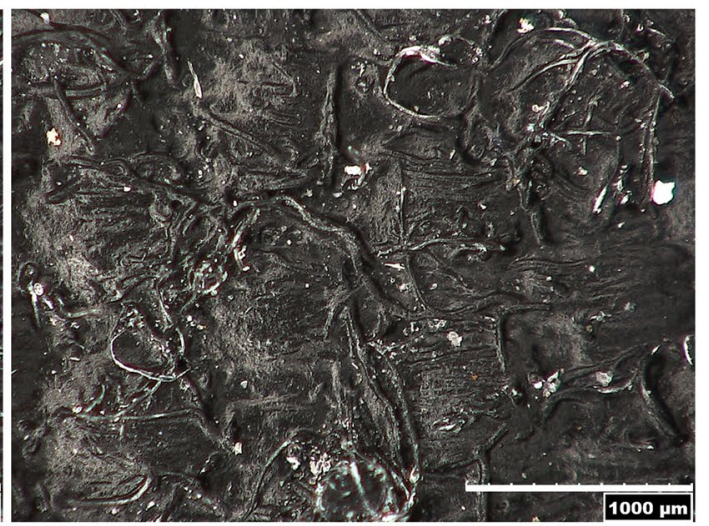

Nanorestore Gel® Peggy 6

BS2 1\% w/w.

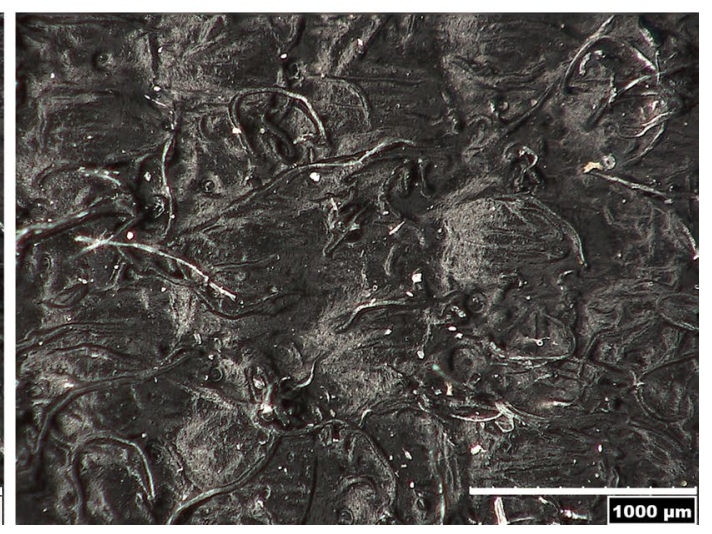

Fig. 11 Digital microscopy (Hirox) images of the black MSA mock-up areas cleaned with Nanorestore Gel ${ }^{\circledR}$ Peggy 5 (on the left) and Nanorestore $\mathrm{Gel}^{\circledR}$ Peggy 6 (on the right) loaded with optimal aqueous cleaning solutions. Scale bar is $1000 \mu$ m; magnification is $\times 100$ 

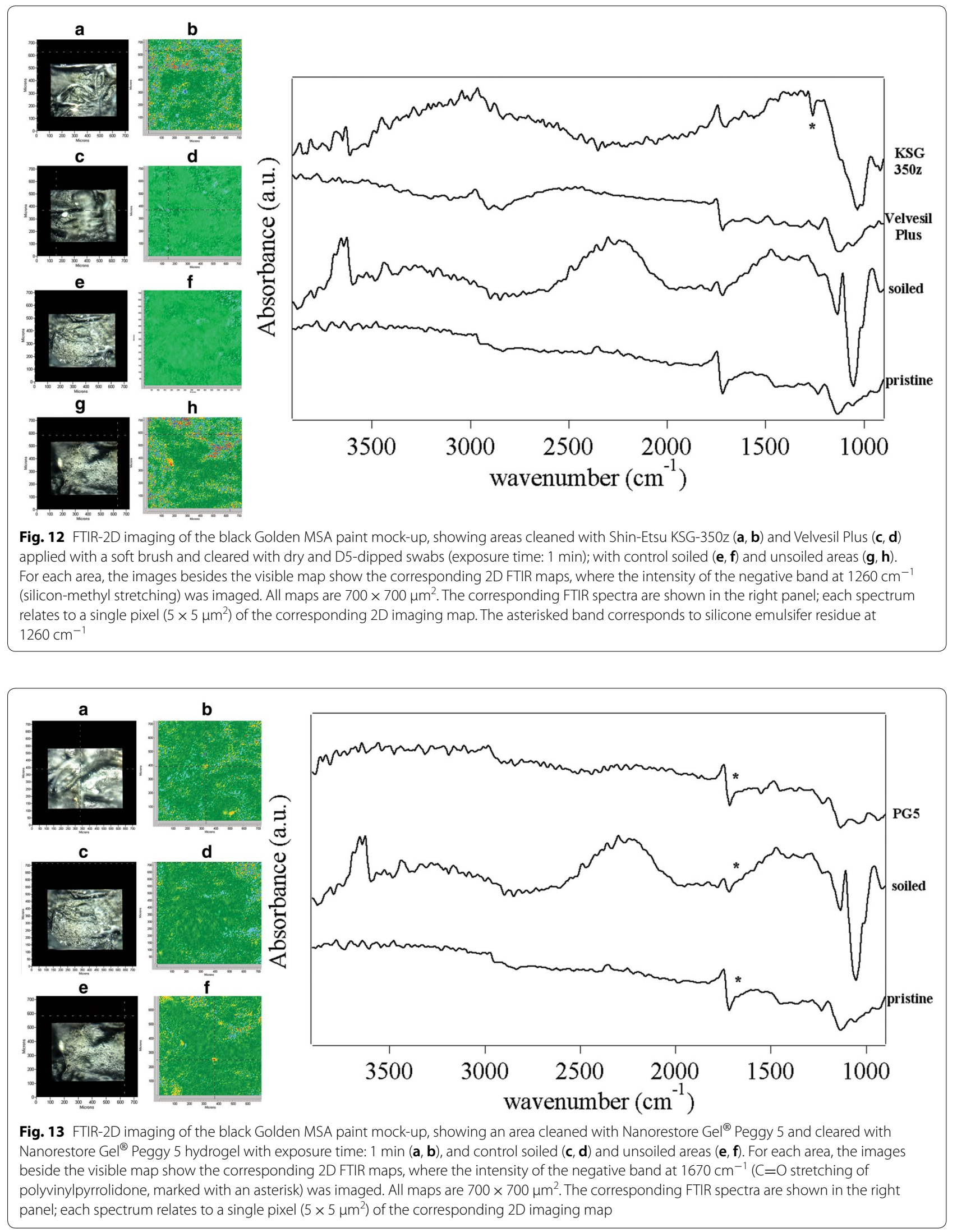


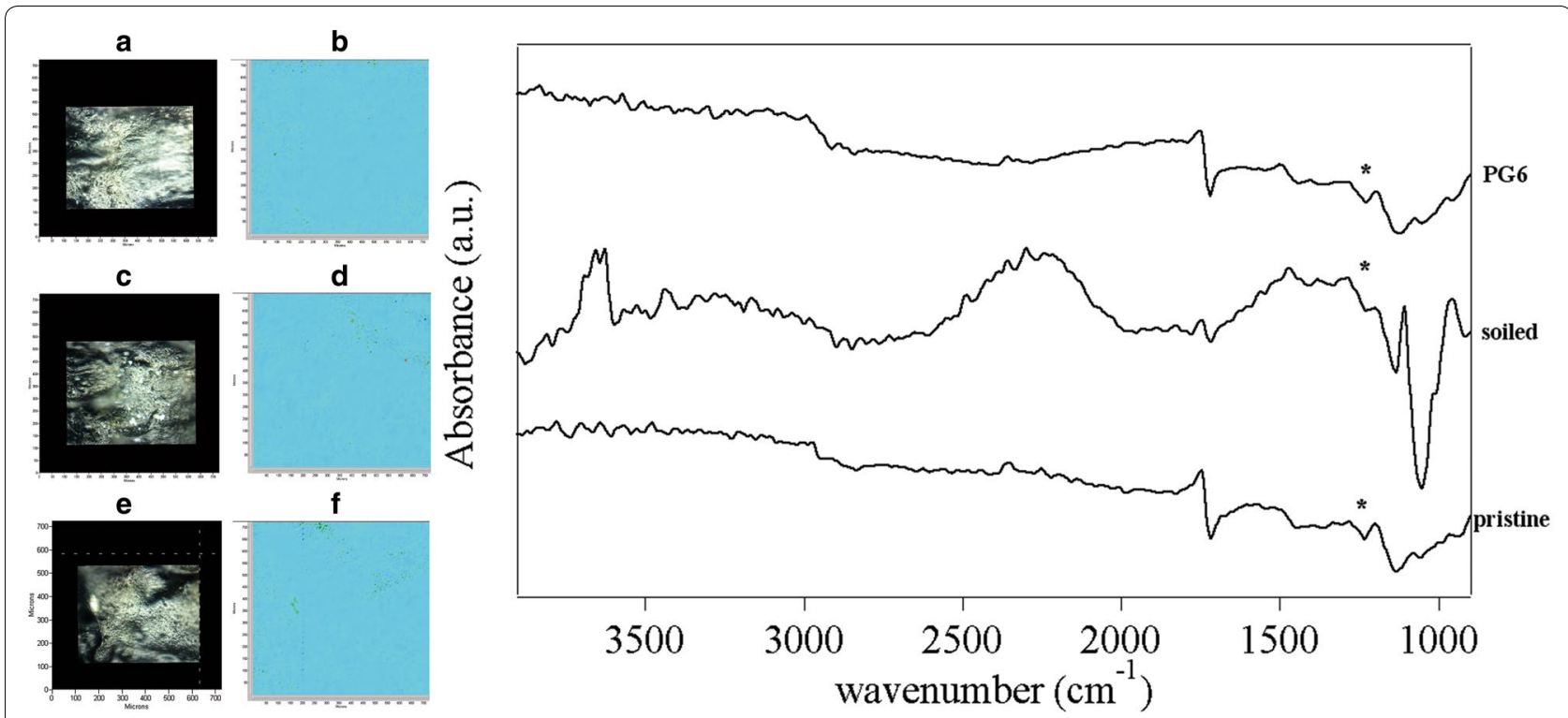

Fig. 14 FTIR-2D imaging of the black Golden MSA paint mock-up, showing an area cleaned with Nanorestore Gel ${ }^{\circledR}$ Peggy 6 and cleared with Nanorestore Gel ${ }^{\circledR}$ Peggy 6 hydrogel with an exposure time: $1 \mathrm{~min}(\mathbf{a}, \mathbf{b})$, and control soiled $(\mathbf{c}, \mathbf{d})$ and unsoiled areas $(\mathbf{e}, \mathbf{f})$. For each area, the images besides the visible map show the corresponding 2D FTIR maps, where the intensity of the negative band at the $1251 \mathrm{~cm}^{-1}$ (C-O stretching of PVA, marked with an asterisk) was imaged. All maps are $700 \times 700 \mu \mathrm{m}^{2}$. The corresponding FTIR spectra are shown in the right panel; each spectrum relates to a single pixel $\left(5 \times 5 \mathrm{~m}^{2}\right)$ of the corresponding $2 \mathrm{D}$ imaging map

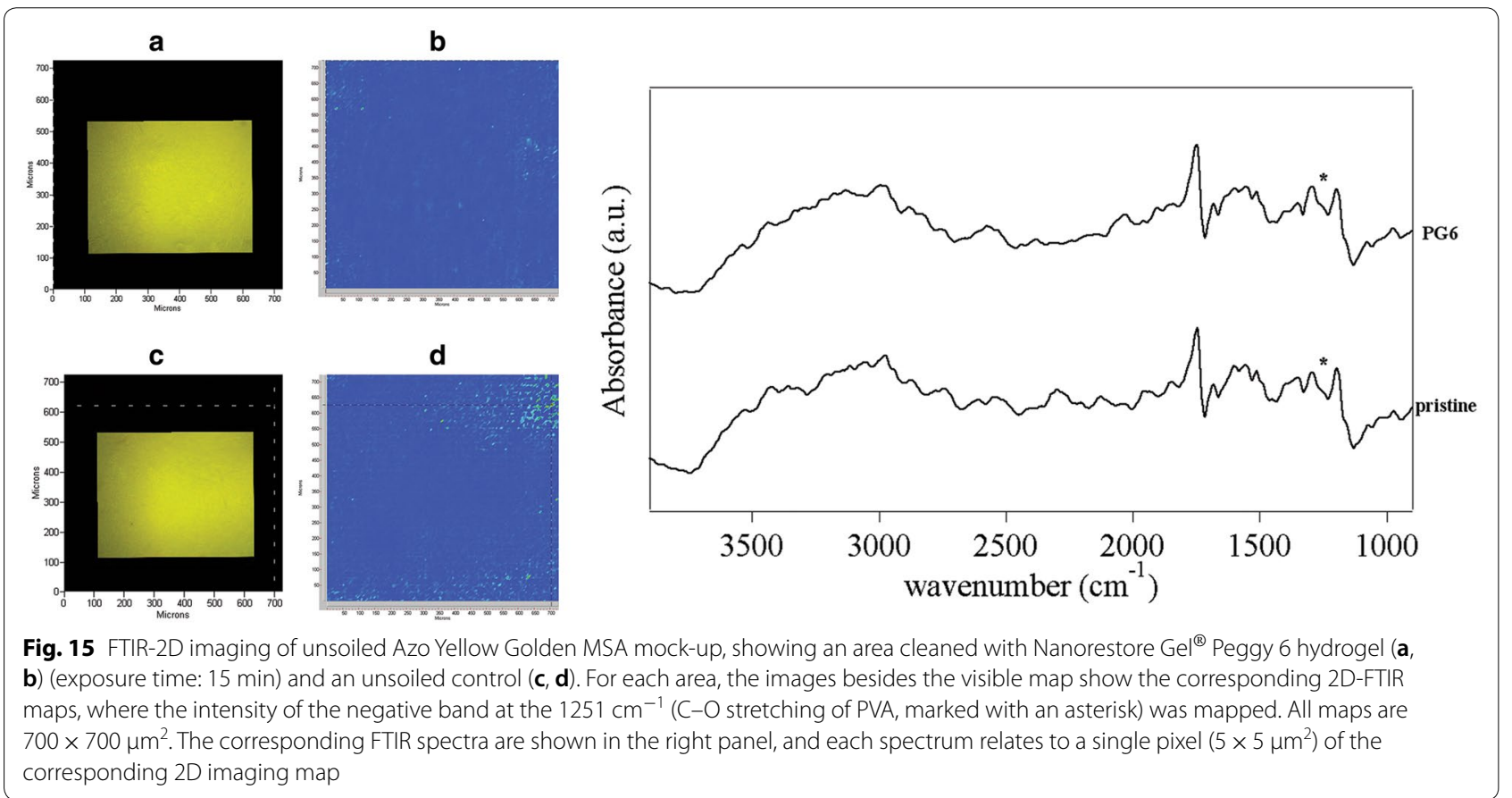

be further minimised through gentle placement and removal of the applied gels, optimal drying of the gels prior to use and minimal manipulation of the gels on the paint surface (Fig. 18).
Discreet tests were also performed on the highly water sensitive cadmium lemon yellow speech bubble area (see Table 1 and Additional file 1: Table S1), using the Nanorestore Gel ${ }^{\circledR}$ Peggy 6 and Nanorestore Gel ${ }^{\circledR}$ Extra Dry (now MWR) hydrogels, where the latter was 


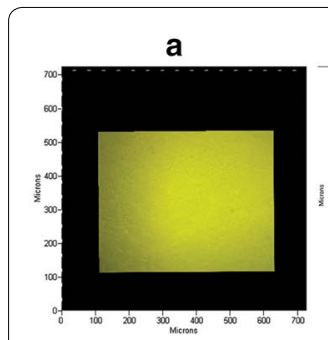

c

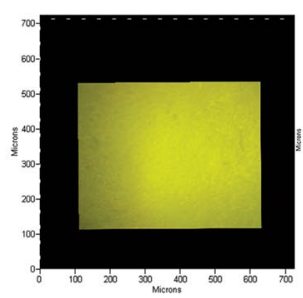

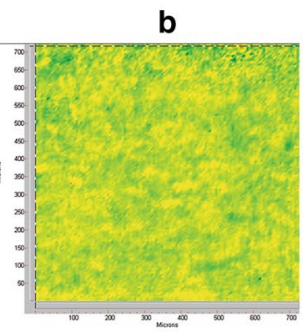

d

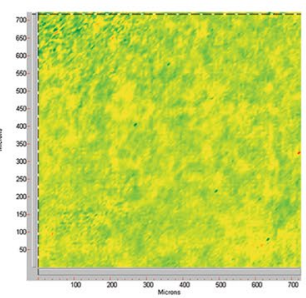

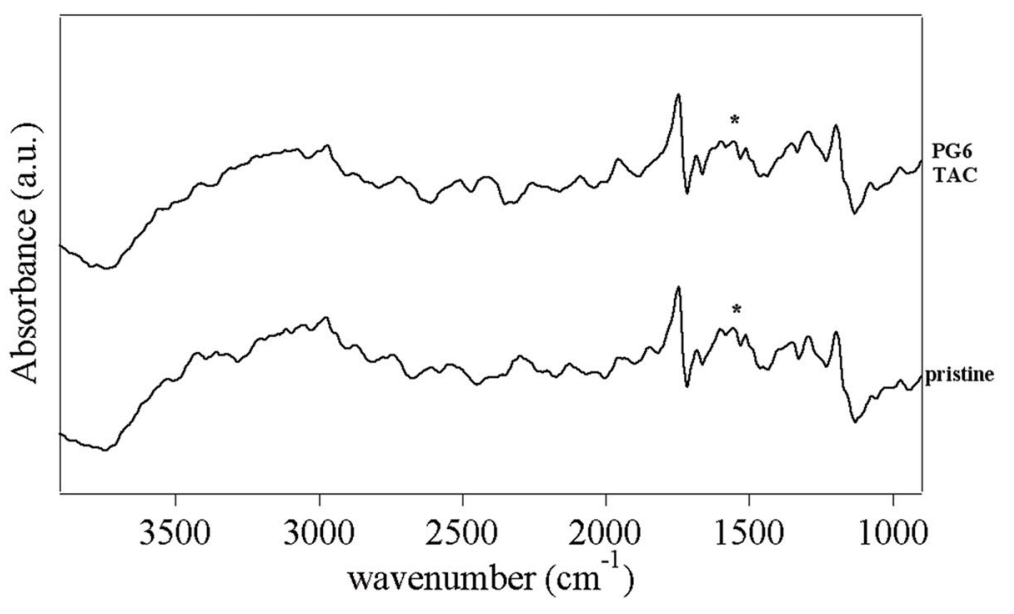

Fig. 16 FTIR-2D imaging of unsoiled Azo Yellow Golden MSA mock-up, showing an area cleaned with Nanorestore Gel ${ }^{\circledR}$ Peggy 6 loaded with tri-ammonium citrate at $1 \%$ w/w $(\mathbf{a}, \mathbf{b})$ followed by a clearance step using a Nanorestore Gel ${ }^{\circledR}$ Peggy 6 hydrogel (exposure time for both steps: $2 \mathrm{~min}$ ) and an unsoiled control (c, d). Possible TAC residues were assessed by mapping the IR symmetric oscillations of the carboxylate ion at $1550 \mathrm{~cm}^{-1}$ (marked with an asterisk). All maps are $700 \times 700 \mu^{2}$. The corresponding FTIR spectra are shown in the right panel. Each spectrum relates to a single pixel $\left(5 \times 5 \mu^{2}\right)$ of the corresponding $2 D$ imaging map

chosen due to its relatively highly water-retentive polymer network. Preliminary results revealed a slight disturbance of the paint surface, including trace levels of pigment pick-up and a light blanching of the surface for both gel types, even for short $45 \mathrm{~s}$ exposures. Given the heterogeneity of the paint layers and the extreme sensitivity to aqueous systems, mineral spirits and mechanical action, wet cleaning treatment was not carried out in this area. ${ }^{24}$

The principal reason for the cleaning treatment was to reduce the visible presence of a yellow-grey dirt layer on the surface, which had accumulated over half a century of display. The dirt had the effect of obscuring the original bright tonality of the painting, as well as exaggerating the glossiness of the surface. The aim the treatment was to minimise the matt-gloss variations induced and/or

\footnotetext{
${ }^{24}$ This area was very heterogeneous, with blended oil and Magna paints, and varying degrees of water, solvent and mechanical sensitivity across the speech bubble area. Trials were carried out using adjusted waters, $\mathrm{pH}$ $5.10 \mathrm{mS} / \mathrm{cm}, \mathrm{pH} 5.2 \mathrm{mS} / \mathrm{cm} ; \mathrm{pH} 6.5,10 \mathrm{mS} / \mathrm{cm}$ and $\mathrm{pH} 8,2 \mathrm{mS} / \mathrm{cm}$, with no improvement regarding pigment pickup. D5 was tested for use as a barrier but, in this case, it did not help to minimise pigment pick-up. Regarding the consequences of not cleaning this area, it is important to emphasise that the speech bubble does not have a flat tonality-there are many modulations in the depth of yellow visible on close inspection. Extensive examination (and analysis - to be detailed elsewhere) revealed that the speech bubble is physically and materially complex. Hence, coupled with its extreme sensitivity to wet cleaning, we decided to leave this area untouched, with the assumption that the soil remaining on this area would not be emphasised after the rest of the painting had been cleaned.
}

enhanced by the soiling.- rather than being true to the variations in saturation of the three main media used: oil (fairly matt), Magna (matt and glossy, depending on numbers of layers) and oil-modified alkyd (matt). While there was a risk involved in not cleaning the speech bubble, the controlled nature of the soiling removal process offered by the Nanorestore ${ }^{\circledR}$ Peggy gels meant we were able (with comprehensive testing) to evenly balance the contrast between the uncleanable (speech bubble) and cleaned areas.

There have been relatively few published examples of Lichtenstein's major works of this period undergoing in-depth cleaning treatments, hence there was also little precedent. The decision to clean Whaam! was taken, in large measure, because the work's visual impact was significantly compromised in its erstwhile condition. So, despite there being little to compare to, with the supporting research undertaken, the risks evaluated and with the new technologies available, we decided to proceed with the treatment of this very sensitive paint surface.

\section{Final protocol for Whaam!}

After the preliminary tests on Whaam! were fully evaluated, the cleaning procedure was optimised and finalised as follows (see Fig. 19):

1. The Nanorestore $\mathrm{Gel}^{\circledR}$ Peggy 6 gels were used as per received dimensions (they were not cut into smaller pieces), as trials exploring the overlapping of gels 


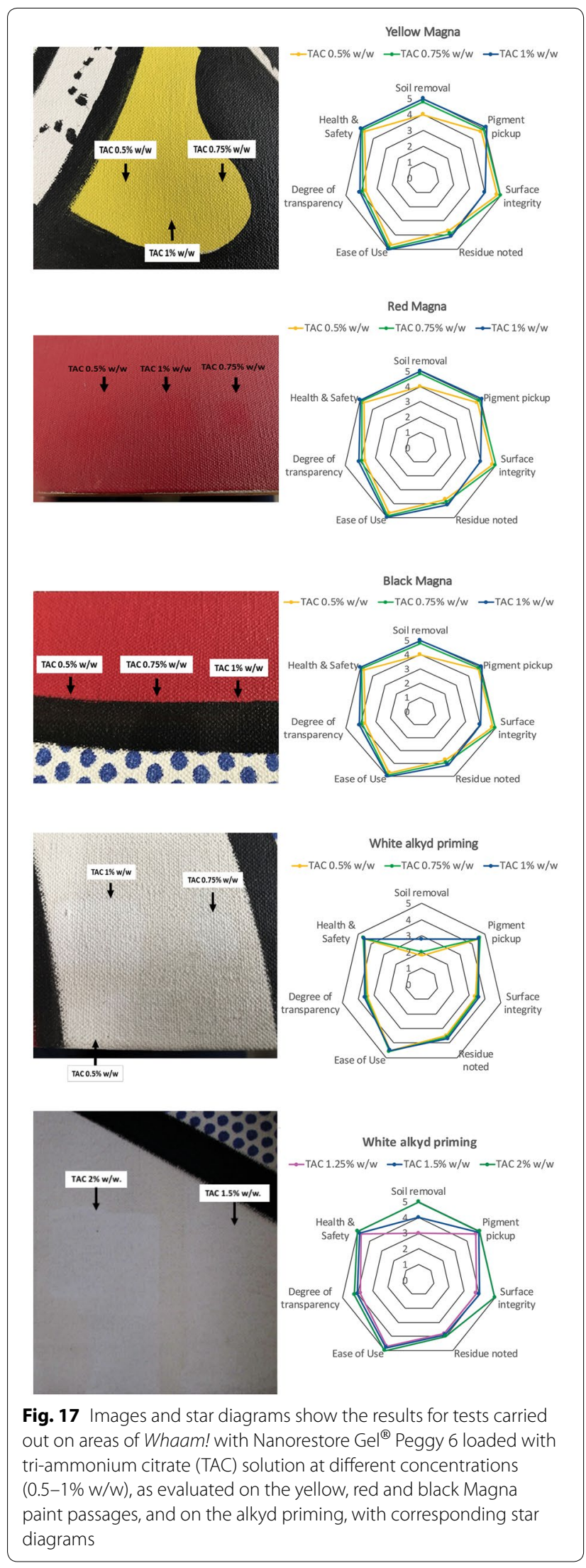

demonstrated that achieving an even cleaning action was possible using the gels as supplied. On occasion, i.e. when cleaning the alkyd priming (requiring higher concentration of TAC) in close proximity to the slightly more TAC-sensitive Magna or oil passages, the gel was applied through a cut-out Mylar template designed to follow the painting composition, in order to protect the surrounding paint surface. $^{25}$

2. Wearing gloves, the already-rinsed (in deionised water) gels were uploaded overnight in the desired $\mathrm{TAC}$ solution, i.e. $0.75 \% \mathrm{w} / \mathrm{w}$ for the Ben-Day dot areas and Magna passages and 2\% w/w for the white alkyd priming.

3. Once ready for use, before application and while wearing gloves, each gel was pressed firmly between a double layer of Evolon ${ }^{\circledR}$ paper to reduce surface and excess liquid. At this stage the gel was very slightly moist to the touch.

4. The gel was then gently placed onto the painting surface and left in place for 2 min (as determined by the trials). During exposure the gel began to dry slightly, resulting in the development of small air-pockets between the underside of the gel and painting surface, hence the gel was very gently pressed using light finger and palm pressure to re-establish contact.

5. After the timed exposure, the gel was gently rolled off the painting surface and placed aside on Evolon ${ }^{\circledR}$ paper for re-application using the other (clean) side of the gel.

6. Immediately after application, a clearance step was performed with Nanorestore Gel ${ }^{\circledR}$ Peggy 6 uploaded with deionised water (hydrogel), applied for the same exposure time. After removal, the surface could be lightly blotted, or left to air dry, as was the case for Whaam!

7. Each gel, after having been used on both sides, was placed into a deionised water bath and soaked for $24 \mathrm{~h}$. The water was changed twice during that time to facilitate the release of imbibed soil. The gels were reused in this way up to 5 times per sheet depending on the degree of soiling imbibed into the gel network. The gel was discarded when it had become too soiled and/or where the physical properties had significantly changed (e.g. tearing when handled or manipulated).

${ }^{25}$ During preliminary testing on Whaam! the possibility of cleaning individual compositional shapes was also considered, in order to avoid tideline formation and potential disturbance of the paint surface when cleaning adjacent areas with different solvent sensitivity. Bigger Nanorestore Gel ${ }^{\circledR}$ Peggy 6 sheets were prepared, of approximately $70 \times 40 \mathrm{~cm}$. However, the sheets were too big to be handled and applied by one person only and had the tendency to break under their own weight. 

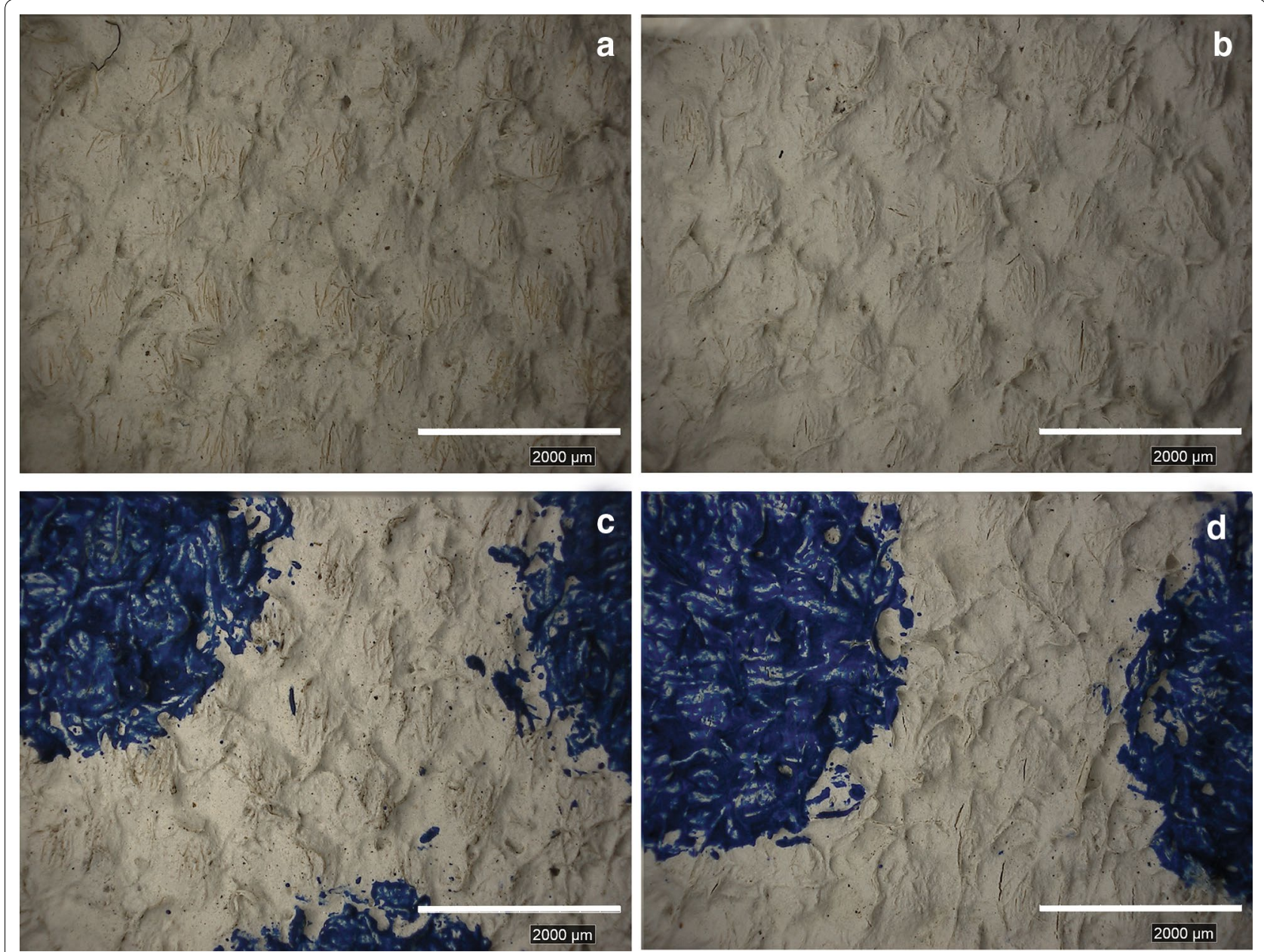

Fig. 18 Digital microscopy (Hirox) images before and after (not at same location) cleaning tests on the alkyd priming (a, b) and blue Ben-Day dot areas (c, d) with Nanorestore Ge ${ }^{\circledR}$ Peggy 6 loaded with a TAC at 2\% and 0.75\% w/w respectively, for a 2-min exposure (followed by the appropriate clearance step). Scale bar is $2000 \mu \mathrm{m}$, magnification is $\times 50$

8. After multiple uses the gels can be dried out (under a fume hood if solvent-such as up to $50 \% \mathrm{v} / \mathrm{v}$ alcohol-has been used) and discarded as regular waste.

Figure 20 includes an image of Whaam! where the cleaning treatment of the left-hand panel (only) has been completed. Here the differences between the two panels are perceived as an overall lighter tone, an enhanced saturation and brightness of colours and greater contrast between the black and white areas of the composition. In total, approximately 150 gels were used to complete the treatment; and once the system had been optimised through the extensive research phase, the soiling removal treatment took approximately 90 days to complete.

\section{Post-cleaning assessment}

Post-treatment assessments confirmed that there were no detectable changes to the paint surfaces other than the removal of the soiling layer. Though the colours, lines and contrast between the light and dark areas of the painting were more evident after treatment, the colour change data (Additional file 1: Figure S3) suggests that the change resulting from the removal of the yellowed soiling was minor, though still detectable. In addition, there were minor gloss changes resulting from the treatment (Additional file 1: Figure S4), with most colours recording small shifts towards a slightly higher gloss value, which presumably now reflects the natural gloss of the paint layers.

\section{Retouching}

While the surface cleaning treatment had successfully revived Whaam!'s sharp, crisp composition, there remained a number of slightly disturbing surface scuffs 


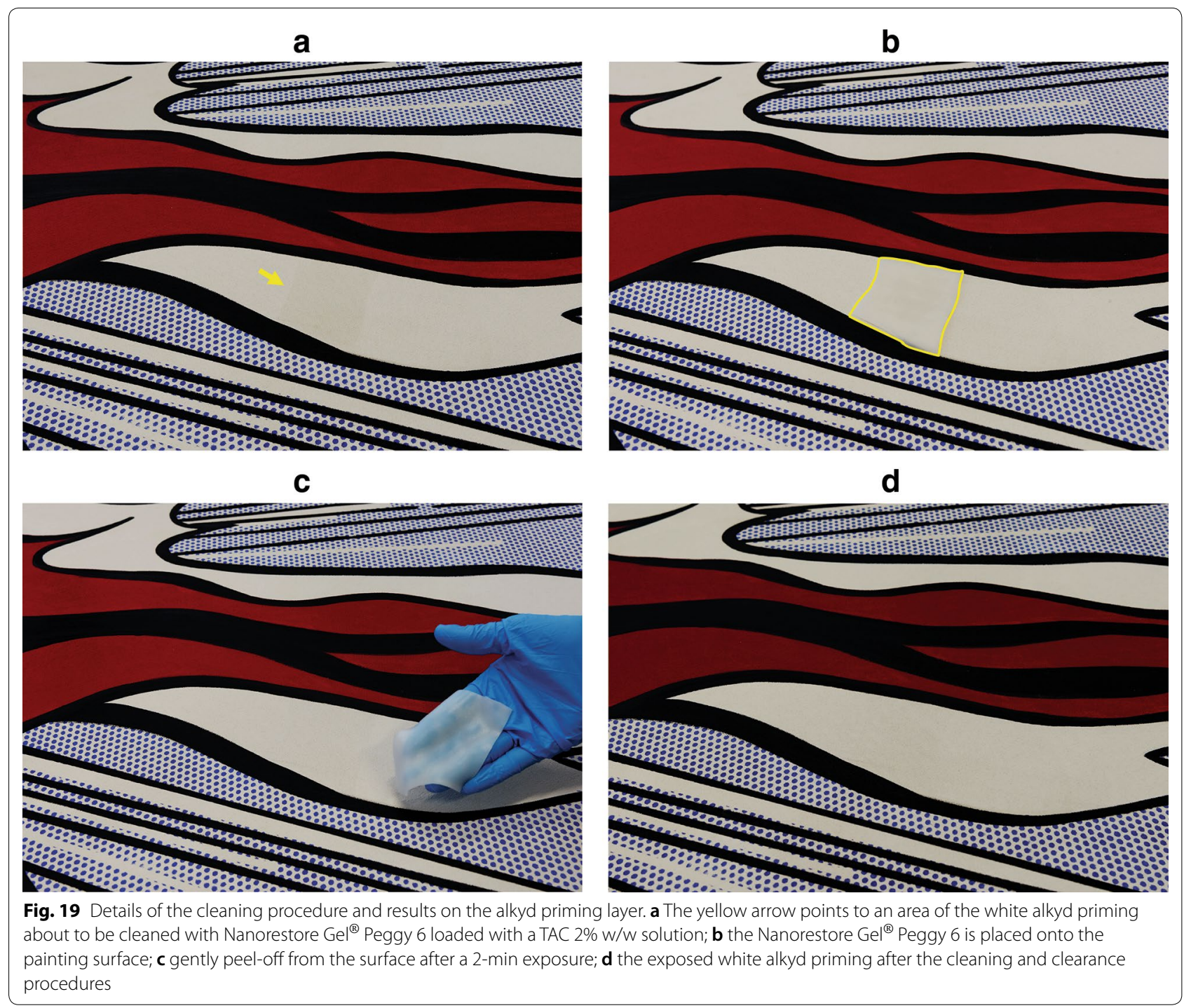

and scratches around the edges of both panels. The discreet but necessary cleaning tests to the surface of the speech bubble also remained slightly visible upon close inspection. As it was therefore necessary to minimise these visual effects-and as the painting was sensitive to both polar and non-polar retouching systems-finding suitable materials (both binder and pigment) proved challenging, as any such system could further disturb the underlying paint and risk becoming permanent. After some initial trials (not described), pastel was chosen for the retouching phase of the treatment [42]. The pastels were pulverised to a fine grind using a mortar and pestle and applied using a sable brush in light strokes. They were then mixed to create the right tone and/or colour to match the original paint as required. As the subsequent bond of the material to the paint surface is relatively weak, the retouching is thus considered temporary and will need to be repeated. This practice is, none the less, preferable to using a more permanent system that cannot be safely reversed. More specifically, combinations of pulverised ochre, chrome and cadmium yellow Schminke pastel ${ }^{26}$ were used to retouch the ingrained finger-marks and discreet cleaning test sites on the yellow speech bubble. Other scuffs and scratches to the Magna colours around the edges were also retouched, and a series of small narrow scratches were integrated using sharpened Museum Aquarelle Caran D'ache water-resoluble pencils. ${ }^{27}$

\footnotetext{
${ }^{26}$ https://www.schmincke.de/en/products/pastels.html.

27 https://store.carandache.com/uk/en/1527-museum-aquarelle?chosen.
} 


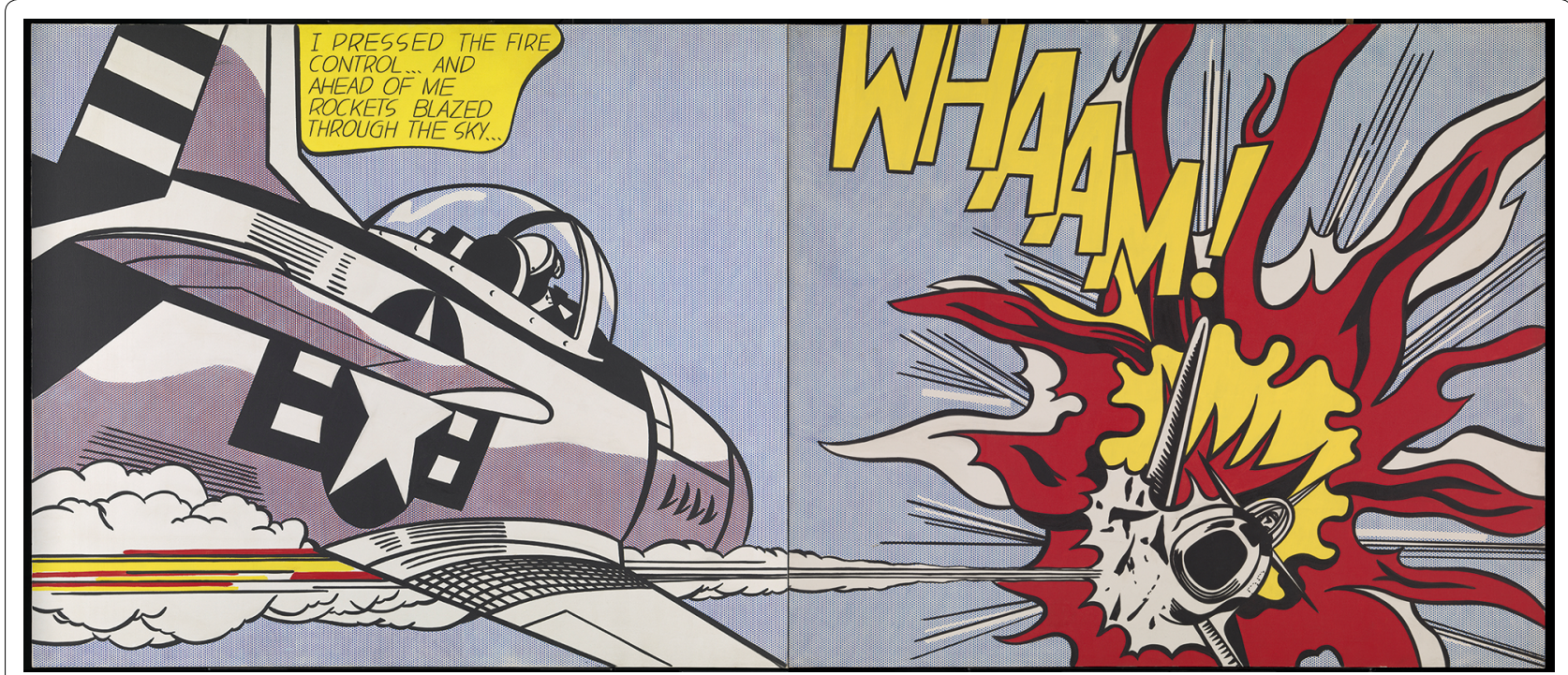

Fig. 20 Whaam! during cleaning. The difference between the left (cleaned) and right (yet to be cleaned) panels is evident. Photo @Tate 2017; @The estate of Roy Lichtenstein

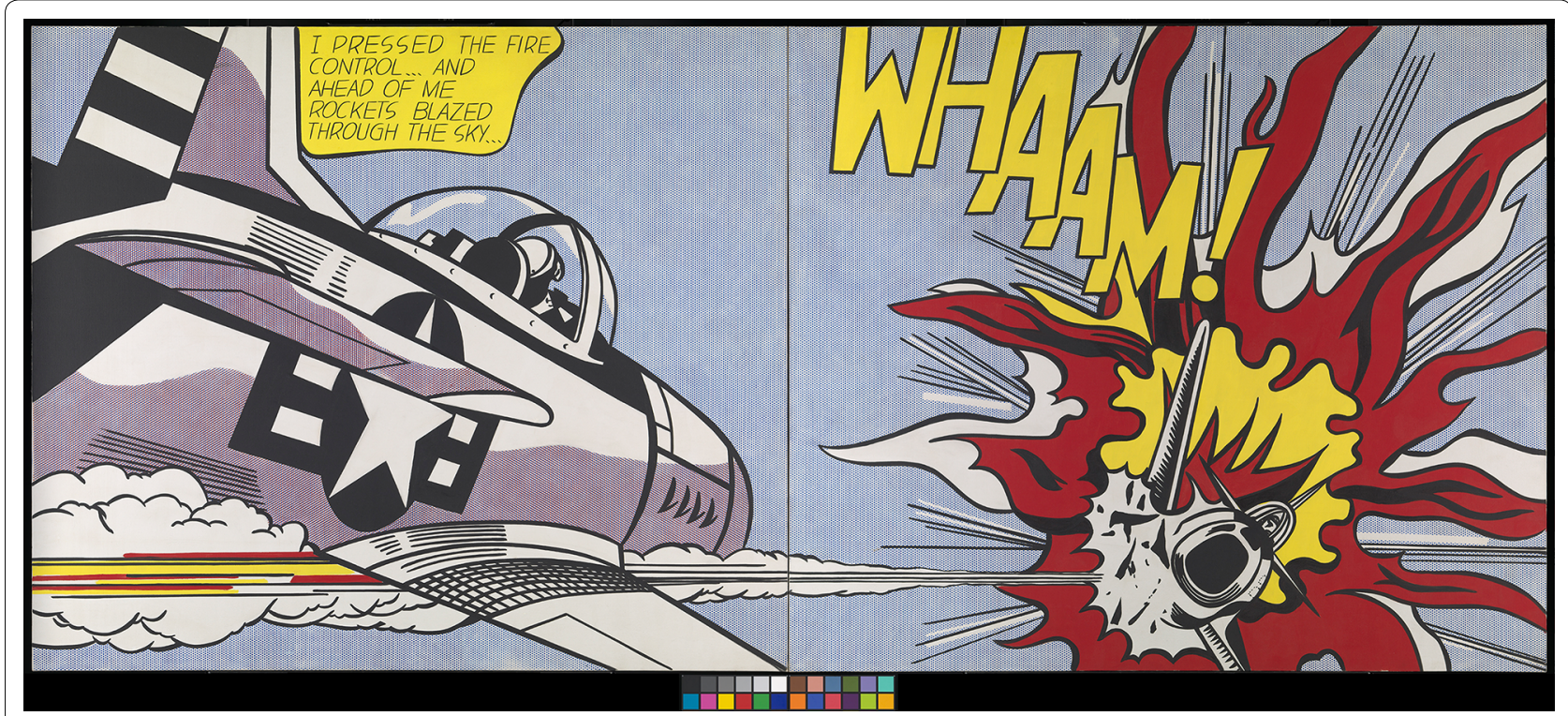

Fig. 21 Roy Lichtenstein's Whaam! after conservation treatment-Tate T00897; Photo @Tate 2018; @The estate of Roy Lichtenstein

\section{Conclusions}

This paper presents rigorous, practice-based research which supported, facilitated and guided the first surface cleaning treatment of Roy Lichtenstein's iconic painting Whaam! (Fig. 21). The methodology employed produced new insights into Lichtenstein's material practice and aesthetic, added to our knowledge of the properties of Magna paints and facilitated a reflective, iterative evaluation of a range of established and novel cleaning systems, several of which were also assessed for possible residues. The process of determining an appropriate cleaning method involved exploring the work of art in detail, assessing the context of the production of the work, exploring the current painting surface and determining the aims of, and the restrictions inherent to, any intended conservation treatment. The treatment optimisation research commenced through exploring which solvents could form the base of wet-cleaning systems, followed by optimising the most appropriate solvents through the control of $\mathrm{pH}$, conductivity, the addition of surfactants and chelators, and controlling their application (to minimise mechanical action) using spreadable, rigid 
and flexible gels as well as silicone emulsifiers. Each step built on previous results, as the research was designed to closely followed the process of conservation treatment decision-making. Along the way, as systems were trialled and evaluated on mock-ups and as discreet tests on the painting, several options were discarded due to unacceptable levels of risk. It is important to note that, even if specific materials were deemed unsuitable for this case study, each of the materials evaluated presented advantages and disadvantages, which may make them better suited to other works of art and conservation challenges.

As the evaluations progressed, the Nanorestore ${ }^{\circledR}$ Peggy series of PVA-based hydrogels-proved highly promising. After optimising the aqueous phase, the Nanorestore ${ }^{\circledR}$ Peggy gels were extensively evaluated through trials on mock-ups; eventually proving to be the most effective and low-risk option for use on Whaam!; though it is noted that the highly-water sensitive speechbubble area proved too sensitive for all available systems. For Whaam!, the Peggy 6 gel was used with controlled concentrations of triammonium citrate (TAC), and as the associated residue studies involved assessing samples for gel and TAC residues only; hence further exploration of these gels used with different solvents and additives is warranted. Nonetheless, the two key advantages offered by these gels, which rendered them most suitable to this case study treatment, included the efficient uptake of soil and the unique flexibility of the Peggy 6 gel in particular, which when combined with the extensive supporting research, enabled the successful treatment of this iconic painting for the first time in the painting's history.

\section{Supplementary information}

Supplementary information accompanies this paper at https://doi. org/10.1186/s40494-020-0350-2.

\footnotetext{
Additional file 1: Figure S1. The completed MSA paint (Magna equivalent) and Ben-Day dot (W\&N oil paint) mock-up panels after the application of the artificial indoor soil. Figure S2. Digital microscopy (Hirox) images of the yellow MSA mock-up acquired for the control unsoiled and soiled areas, and for areas cleaned with the different gels prepared with deionised water only, and corresponding star diagrams. The scale bar is $1000 \mu \mathrm{m}$, magnification is $\times 100$. Figure $\mathbf{S} 3$. Colour change $\Delta \mathrm{E}^{00}$ data after cleaning - left-hand panel (LHP, on the left) and right-hand panel (RHP, on the right). The white alkyd and yellow Magna areas became lighter, with the white alkyd also shifted to a less yellow hue. The red and black Magna areas became darker (i.e. more saturated). Figure S4. Gloss measurement data (at $60^{\circ}$ ) before (orange bars) and after (green bars) cleaning for left-hand panel (LHP, on the left) and right-hand panel (RHP, on the right). Minimal changes in gloss were noted, with most shifts falling within meas urement error. Table S1. Key observations from discrete swab-roll trials with deionised water on Whaam!. Table S2. List of criteria and description of the rating scales used to evaluate free-liquid options on Whaam! Table S3. List of criteria and descriptions of the rating scales used to evaluate the suitability of gels and emulsifiers on the range of mock-up samples and Whaam!. Table S4. Key observations from 10-swab roll trials with free-liquid options on the range of mock-up samples.
}

\section{Abbreviations}

NANORESTART: NANO materials for the RESToration of ART; MOMA: Museum of Modern Art (New York); nBMA: nbutyl methacrylate; PW6: pigment white 6; PB29: pigment blue 29; PR108: pigment red 108; PY34: pigment yellow 34; PY35: pigment yellow 35; PBk11: pigment black 11; PBr8: pigment brown 8; PY3: pigment yellow 3; UV: ultraviolet; FTIR: Fourier transform infrared spectroscopy; SEM: scanning electron microscopy; EDX: energy dispersive X-ray analysis; GCMS: gas chromatography-mass spectrometry; PyGCMS: pyrolysis gas chromatography-mass spectrometry; SCl: spectral component included; SCE: spectral component excluded; GU: gloss units; ATR: attenuated total reflectance; 2D: two dimensional; MSA: mineral spirits acrylic; D5: decamethylcyclopentasiloxane; MES: 2-(N-morpholino)ethanesulfonic acid; AMU: atomic mass units; W\&N: Winsor and Newton; PVA: polyvinyl alcohol; PVP: polyvinyl pyrrolidone; DI: deionised (water); TAC: Tri-ammonium citrate.

\section{Acknowledgements}

The authors would like to acknowledge and thank the following people and institutions: all NANORESTART partners; Tate Conservation and Research Departments-in particular Joyce Townsend, Luigi Galimberti, Pip Laurenson, Deborah Potter, Jane McCree and Annette King; Tate Curatorial Department, in particular Matthew Gale, Gregor Muir and Frances Morris; Tate Photography Department; Tom Learner, Getty Conservation Institute US; Roy Lichtenstein Foundation, US; Mark Golden, Golden Artist Colors US; Chris Stavroudis, private practice US; Sandra Amann, Amman and Estabrook Conservation Associates, US; the three peer reviewers and Richard Brereton.

\section{Authors' contributions}

BO (Tate) designed, led and managed Tate's contribution to the NANORESTART project (2015-2018) with the support of Tate's Research and Conservation Departments. The wider NANORESTART project was led by Professor PB (CSGI) and DC (CSGI) was a project Co-ordinator. AB was the Tate NANORESTART Post-doctoral Conservation Science researcher (2017-2019), LVA was the Tate NANORESTART Post-doctoral Conservation Science researcher (2016-2017). RB was the Tate NANORESTART Conservator for this case study (2017-2018). RB carried out research into the appearance and art historical context of the painting; determined the specific and broader aims of the cleaning treatment and carried out the full conservation treatment. RB, $L V A, A B$ and $B O$ carried out the technical examination of the painting. $A B, B O, J L$ and LVA performed the fundamental paint analysis and data interpretation, with Joyce Townsend (Tate), JL (Tate), DC (CSGI) supporting cross-section and EDX, GCMS, FPA-FTIR analysis and interpretation respectively. RB and LVA prepared the mock-up samples. BO, AB and RB informed the cleaning tests and evaluation methodologies. RB performed the cleaning tests on all mock-ups and the painting. $A B$ and $R B$ documented the evaluation of cleaning materials, $A B$ and $\mathrm{BO}$ managed the acquired data for the evaluation phase and treatment, RB managed the technical and conservation images and documentation. $A B$ supported the conservation treatment process through the preparation and cleaning of gels and the systematic evaluation of the painting surface. RB directed and arranged all technical photography and AB performed all Hirox imaging, colour and gloss measurement. NB (formerly CSGI) synthesised the Nanorestore $\mathrm{Gel}^{\circledR}$ used for the later research phase and conservation treatment. $A B$ produced the first draft of the manuscript and contributed to several edits; $\mathrm{BO}, \mathrm{RB}$ and $\mathrm{DC}$ contributed text to the manuscript, and $\mathrm{AB}$ gathered all images and data for publication. BO fully edited the manuscript. All authors contributed to the manuscript text and/or data presented. $A B, R B, P B, D C$, and $\mathrm{BO}$ approved the manuscript prior to submission and $\mathrm{BO}, \mathrm{AB}, \mathrm{RB}$ and $J \mathrm{~L}$ edited and approved the subsequent versions prior to publication. All authors read and approved the final manuscript.

\section{Funding}

The NANORESTART project received funding from the European Union's Horizon 2020 research and innovation programme under Grant Agreement No 646063. The purchase of Tate's Hirox KH-8700 digital microscope was supported in part by the NANORESTART project. The conservation treatment was also partly funded by Tate.

Availability of data and materials

All the data are available within the manuscript.

Competing interests

The authors declare that they have no competing interests. 


\section{Author details}

${ }^{1}$ Conservation Department, Tate, Millbank, London SW1P 4RG, UK. ${ }^{2}$ Present Address: Department of Conservation and Restoration and Research Unit LAQV-REQUIMTE, NOVA School of Sciences and Technology (FCT NOVA), 2829-516 Monte da Caparica, Portugal. ${ }^{3}$ Present Address: Rachel Barker Associates, London, UK. ${ }^{4}$ Department of Chemistry, University of Florence and Centre for Colloid and Surface Science (CSGI), Via della Lastruccia 3, 50019 Sesto Fiorentino, Firenze, Italy. ${ }^{5}$ Present Address: The National Archives, Kew, Richmond, Surrey, London TW9 4DU, UK.

Received: 4 September 2019 Accepted: 15 January 2020

\section{Published online: 31 January 2020}

\section{References}

1. Waldman D. War comics, 1962-64. Roy Lichtenstein. New York: Solomon R. Guggenheim Museum; 1993. p. 91-109.

2. Crook J, Learner T. Roy Lichtenstein. The impact of modern paints. London: Tate Gallery Publishing Ltd; 2000. p. 112-25.

3. Bailey M. Who opposed a $£ 4,665$ Lichtenstein?. Philadelphia: The Art Newspaper Museum; 2013.

4. Tate Public Records: research: artists: conservation interviews TG 23/1/1/111, Jo Crook interview with Roy Lichtenstein, New York studio 26th May 1992.

5. Ziraldo I, Watts K, Luk A, Lagalante AF, Wolbers RC. The influence of temperature and humidity on swelling and surfactant migration in acrylic emulsion paint films. Stud Conserv. 2016;61(4):209-21.

6. Lagalante A, Wolbers RC. Particle-based silicone cleaning emulsions: studies in model paint systems for the cleaning of water-sensitive artworks. In: Angelova LV, Ormsby B, Townsend JH, Wolbers RC, editors. Gels in the conservation of art. London: Archetype Publications; 2017. p. 193-9.

7. Tate conservation report file for Roy Lichtenstein Whaam! (T00897).

8. Lee J, Bonaduce I, Modugno F, La Nasa J, Ormsby B, van den Berg KJ. Scientific investigation into the water sensitivity of twentieth-century oil paints. Microchem J. 2018;138:282-95.

9. Upright D. Morris Louis: the complete paintings (A Catalogue Raisonné). New York: Harry N. Abrams Inc; 1985. p. 49-58.

10. Gates G, Ausema T, Lake S. What makes the colour field? A technical examination of Magna paint. In: Learner TJS, Smithen P, Krueger J, Schilling MR, editors. Modern paint uncovered. Los Angeles: Getty Publications; 2007. p. 277-8.

11. Rogge CE, Epley B. Behind the Bocour label: identification of pigments and binders in historic Bocour oil and acrylic paints. J Am Inst Conserv. 2017:56(1):15-42.

12. Skalka M. Personal communication (email) with B. Ormsby, 4.6.2019.

13. Berg JDJ, Berg KJ, Boon JJ. Chemical changes in curing and ageing oil paints. In: 12th triennial meeting Lyon 29 August-3 September 1999, vol. 1, ICOM-CC ICOM Committee for Conservation, James. London: James and James (Science Publishers); 1999. p. 248-53.

14. Tempest H, Burnstock A, Saltmarsh P, van den Berg KJ. Sensitivity of oil paint surfaces to aqueous and other solvents. In: Mecklenburg MF, Charola AE, Koestler RJ, editors. New insights into the cleaning of paintings: proceedings from the cleaning 2010 international conference, Universidad Politecnica de Valencia and Museum Conservation Institute. Washington, DC: Smithsonian Institution (Smithsonian Contributions to Museum Conservation); 2013. p. 107-14.

15. Silvester G, Burnstock A, Megens L, Learner T, Chiari G, van den Berg K. A cause of water-sensitivity in modern oil paint films: The formation of magnesium sulphate. Stud Conserv. 2014;59(1):38-51.

16. Wolbers $R$. The use of a synthetic soiling mixture as a means for evaluating the efficacy of aqueous cleaning materials on painted surfaces. Bulletin of the Association des Restaurateurs d'Art et d'Archéologie de Formation Universitaire (ARAAFU): Conservation-Restauration des Biens Culturels. 1992; 4:22-29.

17. Ormsby BA, Soldano A, Keefe MH, Phenix A, Learner T. An empirical evaluation of a range of cleaning agents for removing dirt from artists' acrylic emulsion paints. AIC Paint Spec Group Postprints. 2013;11:77-87.
18. Stavroudis C, Doherty T. The modular cleaning program in practice: application to acrylic paintings. In: new insights into the cleaning of paintings: proceedings from the cleaning 2010 international conference. Universidad Politecnica de Valencia and Museum Conservation Institute; 2013. p. 139-45.

19. Dillon CE, Lagalante AF, Wolbers R. Acrylic emulsion paint films: the effect of solution $\mathrm{pH}$, conductivity, and ionic strength on film swelling and surfactant removal. Stud Conserv. 2014;59(1):52-62.

20. Sorano-Stedman V, Wolbers R, Germond S. The treatment of Barnett Newman's shining forth in the MNAM Pompidou collection. In: Angelova LV, Ormsby B, Townsend JH, Wolbers R, editors. Gels in the conservation of art. London: Archetype Publications Ltd; 2016. p. 29-35.

21. Stavroudis C. Gels: evolution in practice. In: Angelova LV, Ormsby B, Townsend JH, Wolbers R, editors. Gels in the conservation of art. London: Archetype Publications; 2017. p. 209-17.

22. Stavroudis C. More from CAPS3: surfactants, silicone-based solvents, and microemulsions. WAAC Newsl. 2012;34(3):24-7.

23. Doherty T, Shockey L. The use of siloxane-based systems to clean watersensitive painted surfaces. WAAC Newsl. 2013;35(2):15-7.

24. Lagalante AF, Wolbers RC. The cleaning of acrylic paintings: new particle-based water-in-oil emulsifiers. In: Selva Bonino V, editor. Dall'Olio All'Acrilico, Dall'Impressionismo all'Arte Contemporanea, 7th international congress, Milano 13-14 November 2015; 2016. p. 107-14.

25. lannuccelli $S$, Sotgiu S. Wet treatments of works of art on paper with rigid gellan gels. Book Pap Group Annu. 2010;29:25-39.

26. Casoli A, Cremonesi P, Isca C, Groppetti R, Pini S, Senin N. Evaluation of the effect of cleaning on the morphological properties of ancient paper surface. Cellulose. 2013;20(4):2027-43.

27. Skelton S, Rogge C, Bomford ZV. Testing the limits: the theoretical development and practical reality of a large-scale agarose gel treatment for a discolored Morris Louis. Stud Conserv. 2016;61(sup2):214-8.

28. Diamond $O$, Barkovic M, Cross M, Ormsby B. The role of agar gel in treating water stains on acrylic paintings: case study of composition, by Justin Knowles. J Am Inst Conserv. 1963;2019:1-14.

29. Chelazzi D, Giorgi R, Baglioni P. Microemulsions, micelles, and functional gels: how colloids and soft matter preserve works of art. Angew Chem Int Ed. 2018:57:7296-303.

30. Domingues JAL, Bonelli N, Giorgi R, Fratini E, Gorel F, Baglioni P. Innovative hydrogels based on semi-interpenetrating $\mathrm{P}(\mathrm{HEMA}) / \mathrm{PV} P$ networks for the cleaning of water-sensitive cultural heritage artefacts. Langmuir. 2013;29(8):2746-55

31. Domingues J, Bonelli N, Giorgi R, Fratini EBP. Innovative method for the cleaning of water-sensitive artifacts: synthesis and application of highly retentive chemical hydrogels. Int J Conserv Sci. 2013;4:715-22.

32. Domingues J, Bonelli N, Giorgi R, Baglioni P. Chemical semi-IPN hydrogels for the removal of adhesives from canvas paintings. Appl Phys A Mater Sci Process. 2014;114(3):705-10.

33. Nunberg S, Alcala S, Tomkiewicz C, McGlinchy C, Henry M, Dittmer J. Conservation of a White Louise Nevelson installation: gel systems explored. In: Angelova LV, Ormsby B, Townsend JH, Wolbers R, editors. Gels in the conservation of art. London: Archetype Publications Ltd; 2017. p. 299-305

34. Eriksson H, Wedberg I, Nesson J, Bronmark-Thourlund M. The use of nanorestore gels in the conservation of lime-based wall-paintings. In: Angelova LV, Ormsby B, Townsend JH, Wolbers R, editors. Gels in the conservation of art. London: Archetype Publications Ltd.; 2017. p. 270-3.

35. Baglioni P, Baglioni M, Bonelli N, Chelazzi D, Giorgi R. Smart soft nanomaterials for cleaning. Nanotechnologies and nanomaterials for diagnostic conservation and restoration of cultural heritage. Amsterdam: Elsevier; 2019. p. 171-204.

36. Baglioni P, Carretti E, Chelazzi D. Nanomaterials in art conservation. Nat Nanotechnol. 2015;10:287-90. https://doi.org/10.1038/nnano.2015.38.

37. Bonelli N, Montis C, Mirabile A, Berti D, Baglioni P. Restoration of paper artworks with microemulsions confined in hydrogels for safe and efficient removal of adhesive tapes. Proc Natl Acad Sci. 2018;115(23):5932-7. https ://doi.org/10.1073/pnas.1801962115.

38. Mastrangelo R, Montis C, Bonelli N, Tempesti P, Baglioni P. Surface cleaning of artworks: structure and dynamics of nanostructured fluids confined in polymeric hydrogel networks. Phys Chem Chem Phys. 2017;19(35):23762-72. 
39. Bonelli N, Poggi G, Chelazzi D, Giorgi R, Baglioni P. Poly(vinyl alcohol)/ poly(vinyl pyrrolidone) hydrogels for the cleaning of art. J Colloid Interface Sci. 2019;536:339-48.

40. Chung JY, Ormsby B, Lee J, Burnstock A, Van den Berg KJ. An investigation options for surface cleaning unvarnished water-sensitive oil paints based on recent developments for acrylic paints. In: ICOM-CC 18th triennial conference preprints, Copenhagen, 4-8 September 2017. Paris: International Council of Museums; 2017.

41. Ormsby B, Lee J, Bonaduce I, Lluveras-Tenorio A. Evaluating cleaning systems for use on water sensitive modern oil paints: a comparative study. In: van den Berg KJ, Bonaduce I, Burnstock A, Ormsby B, Scharff M, Carlyle L, Heydenreich G, Keune K, editors. Conservation of modern oil paintings. New York: Springer International Publishing; 2019.

42. Choi S, Makin J. Treatment and housing techniques for pastel paintings on paper: case studies. Book Pap Group Annu. 2013;32:33.

\section{Publisher's Note}

Springer Nature remains neutral with regard to jurisdictional claims in published maps and institutional affiliations.

\section{Submit your manuscript to a SpringerOpen ${ }^{\circ}$ journal and benefit from:}

- Convenient online submission

- Rigorous peer review

- Open access: articles freely available online

- High visibility within the field

- Retaining the copyright to your article

Submit your next manuscript at $\boldsymbol{\nabla}$ springeropen.com 